${ }^{+}$Russian State University for the Humanities; amilitarev@gmail.com

‡ Institute of Slavic studies of the Russian Academy of Sciences; sergenicko@mail.ru

\title{
Proto-Afrasian names of ungulates in light of the Proto-Afrasian homeland issue ${ }^{1}$
}

\begin{abstract}
In this paper, we present the first section of a comprehensive thesaurus of Proto-Afrasian zoonyms, compiled and reconstructed by the authors. The list contains more than a hundred names of ungulates, including bovids, pigs, equids and camels, as well as large herbivores (elephants, hippopotamuses and rhinoceroses), allowing for a wholesome (at the current level of our knowledge) picture of these species of fauna in the period preceding the split of Proto-Afrasian (ca. 11th millennium BCE, according to glottochronology) within the area presumably inhabited by speakers of Proto-Afrasian. The results of the reconstruction may be interpreted in favor of two different points of view on the localization of the ProtoAfrasian homeland, namely, the Levant vs. East Africa; the paper presents arguments both in favor of and against each of these hypotheses.
\end{abstract}

Keywords: Proto-Afrasian homeland; Afrasian (Afro-Asiatic) languages; etymology of zoonyms; reconstruction of cultural lexicon.

Until recently, debates about the primary habitat of the speakers of Proto-Afrasian (Afroasiatic, Semito-Hamitic) have been based either upon general and extra-linguistic argumentation, sometimes accompanied with scarce, incomplete and unrepresentative lexical comparisons; or upon the reconstruction of Proto-Afrasian (PAA) terms interpreted as arguments in favor of intensive gathering of wild cereals and beans (Diakonoff 1981) or in favor of proto- or incipient agriculture (Militarev 2002).

It goes without saying that the Urheimat issue cannot be seriously discussed without a complete and detailed reconstruction of PAA animal and plant names, as well as general environmental and cultural vocabularies. Likewise, of vital importance here are the approximate dates of branching of PAA and its descendant proto-languages, obtained by glottochronology. A trustworthy solution of the problem can be achieved only through correlation of comparative linguistic data with the findings and outcomes of such related sciences as archaeology, paleoclimatology, paleontology, and human population genetics.

The genealogical classification of the Afrasian macrofamily as worked out by Alexander Militarev, based first and foremost on lexicostatistics, is as follows (the figures below refer to the approximate BCE dates of branching of proto-languages, obtained glottochronologically by applying Sergei Starostin's recalibrated $\operatorname{method}^{2}$ ):

${ }^{1}$ Alexander Militarev is responsible for all lexical material, reconstruction of zoonyms, and discussion of the hypothetical Levant homeland of Proto-Afrasians and their identification with Natufians. Sergei Nikolaev is responsible for the extra-linguistic evaluation of PAA zoonyms, their paleozoological interpretation, and discussion of the hypothetical African homeland of Proto-Afrasian. We would also like to extend our gratitude to Anastasia Kirillova for her help in compiling the lists of references, the alphabetic index of reconstructed roots, and the list of abbreviations.

Militarev’s research is supported by The Russian Science Foundation (Project № 20-18-00159); the financing organization is The Institute of Linguistics, Russian Academy of Sciences.

${ }^{2}$ As described in, e.g., Starostin 2000. 
Proto-Afrasian - ca. 10,500:

1. Proto-Semitic-Egyptian-Berber-Chadic (PSEBC) - ca. 9,000.

1.1. Proto-Semitic - ca. 4,500 .

1.1.1. Proto-South Semitic (Proto-MSA).

1.1.2. Proto-North Semitic - ca. 3,700.

\subsubsection{Akkadian.}

1.1.2.2. Proto-Central Semitic (Ethiopian-Arabic-Levantine) - ca. 3,000.

1.2. Proto-Egyptian-Berber-Chadic - ca. 7,800.

1.2.1. Egyptian.

1.2.2. Proto-Berber-Chadic - ca. 6,500.

1.2.2.1. Proto-Berber - ca. 1,000 .

1.2.2.2. Proto-Chadic - ca. 5,500.

2. Proto-Cushitic-Omotic (PCO) - ca. 8,800.

2.1. Proto-Cushitic - ca. 7,500.

2.1.1. Proto-North-Central Cushitic - ca. 6,800.

2.1.1.2. Proto-North Cushitic (Beja).

2.1.1.3. Proto-Central Cushitic (Agaw) - ca. 2,000.

2.1.2. Proto-East Cushitic (incl.Yaaku-Mogogodo) - ca. 6,000.

2.1.3. Proto-South Cushitic (incl.Dahalo and Ma'a) - ca. 4,800.

2.2. Proto-Omotic - ca. 6,000.

2.2.1. Proto-North Omotic (incl. Dizi and Mao) - ca. 4,200.

2.2.2. Proto-South Omotic (incl. Ongota) - ca. 4,600.

Until now, any systematic paleozoological argumentation relying on a complete set of PAA animal names has been conspicuously lacking. Today, however, over a hundred names for animals reconstructed by Militarev (who has also drawn from the most reliable comparanda and reconstructions by Olga Stolbova, Aharon Dolgopolsky, Václav Blažek and Gabor Takács, as well as, naturally, those included in SED II) represent terms that can be labeled Proto-Afrasian and refer to species which may be relevant for addressing the problem of the PAA habitat from the point of view of both biocenosis and food acquisition (hunting, fishing, and domestication of animals).

The animal section which is by far the largest (some fifty items), the most economically vital for the Epipaleolithic man of Southwest Asian and North African areas, and one of the most essential for dealing with the issue of the original homeland, consists of PAA names of ungulates (bovids, camels, equids), conventionally labeled as "largest herbivores".

Below we present the complete list ${ }^{3}$ of reconstructed forms ${ }^{4}$ on various levels, together with their reflexes in individual languages.

\section{Bovids and pigs}

1.1a. *2ar(a)w/y-'k. of larger bovid'

${ }^{3}$ Regrettably, we did not have time to include data from the forthcoming Issue VI (Labials) of the Chadic Lexical Database by Olga Stolbova.

${ }^{4}$ It must be stressed that, since historical vocalism in Afrasian (and even Semitic) comparative linguistics is still a weak point, our reconstruction of specific vowels in roots and stems on all levels is in many cases tentative and sometimes rather arbitrary. 


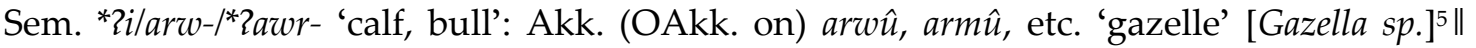

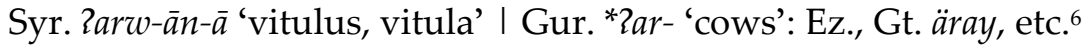

(?) Egyp. (MK) ir.t 'calf', (Dem.) iry.t 'milking cow' (if < 2 ?Vr- not *2Vl-).

Chad. *2arw- 'k. of bovid' [Tragelaphus?]: C. *Zarw-: Baldiu érèz-è 'antelope sp.' || E. *Zar-: Migama 2ấr-ó 'duiker' [Tragelaphus sp.].

Cush. *2araw- 'male of large ungulates': N. *-rayVw- ( ${ }^{*} 2>0$ in Beja): Beja o-reo 'bull, steer' (o- is a masc. article) || E. *2awar- (met.) 'bull': SA: Saho awr, Afar awur 'bull' I LEC: Somali awr 'he-camel', Rendille or 'he-camel, bull', Boni oor 'male elephant' [L. africana], Oromo oor-oo 'burden camel', Bayso aar, Arbore 2aar, Dasenech ar 'bull' I HEC *2aray-: Burji array, arzáy 'bull', ąre 'calf' | Yaaku erer 'antelope sp.' || S. *2arw-: Ma'a áro 'large herbivore' | Dahalo Záároole (with -l root extention?) 'eland' [Taurotragus sp.].7

- Cf. SED II No.16; Blažek 2003b: 236, 269; Mil. 2009; AADB 2476.

1.1b. $2 a r(a) w / y$ - 'k. of lesser bovid'

Sem. *2arwiy- 'ibex' [Capra ibex]: Sab. Rrwy-n pl. '(female) mountain goat, ibex (?)' [Capra ibex] I Arab. 2urw-iyy-at- 'chèvre de montagne' [C. ibex] II MSA: Mhr. 2ar-it 'goat', Jib. Zarü 'she-goat' (Nakano 1986), Mhr. hạ-ráw-n, Hrs. ḥe-werū-n (met.), Jib. Rerú-n 'goats', Soq. Zére-hon 'moutons, chèvres'. ${ }^{8}$

Berb.-Can.: Tenerife ara 'goat'.

Chad. *2awr- (met.) 'small cattle': W. ${ }^{*}(2 a-)$ war-: Hausa ara-ara (-w- lost in a reduplicated form?) 'type of long-legged ram, goat, sheep', Montol, Ankwe, Gerka ur, Kulere wàr 'he-goat' \| E. *2awr-: Lele ōr-ē 'goats'.

Cush. *2aray- 'k. of lesser bovid':9 E. *2aray-: SA *2Vray-: Afar eráy-taa 'goat' I LEC *2ar-: Somali ari, eri 'pecore e capre', Bayso arer 'antelope sp.' I HEC *2aray-: Burji aráy 'sheep' I Yaaku erer 'antelope sp.' || S. *2ary-: Iraqw ari 'goat', pl. ar-a, Alagwa, Burunge ar-a (pl.) 'goats' । Qwadza ali-to $\left(-l-<^{*}-r-\right)$ 'goat'.

- Cf. SED II No. 18; AADB 2447.

\section{2. *2ayl- 'k. of lesser bovid'}

Sem. *2ayil- 'ibex [Capra ibex]': (?) Akk. al-u (el-u) 'a fine breed of sheep' (reading arguable) \| Ugr. 2al, Zil 'ram of superior quality' | Hbr. Zayil 'ram' I ESA: Sab. Zyl 'mountain goat, ibex' [C. ibex], Min. zyl 'bélier, bouquetin' [C. ibex] I Arab. 2iyyal-, zuyyal- 'bouquetin, bouc de montagnes' [C. ibex $]^{10}$

${ }^{5}$ Presumable scientific zoological meanings are quoted in square brackets.

${ }^{6}$ Also Tgr. Zarw-ät (with f. suff. -ät) 'female elephant' [Loxodonta africana] quoted in SED II No. 17 *2arway'wild beast; lion'. For discussion on Gz. 2arwe 'animal, wild animal, beast' with debatable etymological connections, see SED II: 27 (No. 18); on Amh. awra 'male (animal), bull' and its probable areal connections with E. Cush. *2aw(a)r- 'bull' with uncertain direction of borrowing, see SED II: 23 (No. 16). Cf. also Tgr. wuhar 'bull'.

${ }^{7}$ Omot. N.: Oyda arr 'cow' is isolated, likely from E. Cush.

${ }^{8}$ Cf. Sem. *2arn- (likely related with a fossilized $-n$ ) 'k. of antelope or mountain goat': (?) Akk. (MB on) arm-u

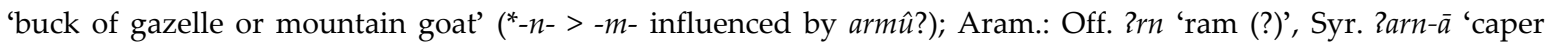
montanus' [C. ibex]; Arab. 2irān- 'oryx mâle' [Oryx sp.] (otherwise <*2araw-); Gz. 2orn-ā 'kind of antelope', Gur.:

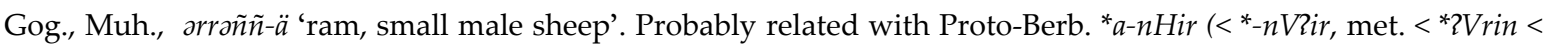
*?Vry-Vn also with a fossilized $-n$ ?): Semlal anìr 'antilope', Zenaga e-na?rh 'gazelle-Dama' [Nangerdama], Ghat inir, Ahaggar enir, Tadghaq ti-nhir-t 'antilope mohor' [N. dama].

${ }^{9}$ Omot. N.: Koyra aar-e 'flock of sheep', Male war-i 'goat' are isolated terms, likely lws. from E. Cush.

${ }^{10}$ Tna. Zil- $\ddot{a}$ 'kind of ram (from the Western Lowlands)' isolated in Eth. is probably a Beja lw.; Jib. ayyól 'Steinbock' [Capra ibex] (Bittner 1917: 29, not in JJ) is probably an Arabism. Cf. also Proto-Sem. *2ayyal- 'Cervus elaphus' (SED II No. 25), similar to PIE *h $h_{1} e l$ - 'deer': Akk. ayal-u 'stag, deer', Ugr. 2ayl 'deer', Phoen. 2yl 'stag' [Cervus 
Berb. *ti-halay (<*-2ayal, met.?) 'sheep': Nefusa t-ili | Zenaga t-ǐ̌i | Ahaggar té-helé, Ghat či-hali, Ayr t-ele, E. Wlm. te-hale, etc. ${ }^{11}$

Cush. *2ayl-'k. of antelope (Litocranius, Tragelaphus, Alcelaphus): N. *2all-i: Beja all-i, pl. ill-i 'long-haired sheep' || E.: SA *2ill-: Saho ill-e, Afar ill-i 'small cattle' | LEC *2el- (< *il- or *2ayl-): Somali eel-o 'tipo de gazella (antilopegiraffa)' [Litocranius walleri], Arbore Rell-ém, Elmolo Rél-em 'ram' || S. *2ayl-: Gorowa ele-te-mo 'bushbuck' [Tragelaphus sylvaticus] I Ma'a izal-é 'ram', izal-ú (met. <*2ayl-?) 'sheep' I Dahalo Rèel-e 'hartebeest' [Alcelaphus buselaphus].

$\Delta$ Egyp. (NE) iy? (if < ${ }^{*} V y y l-$ ) 'Widder' (presumably > Copt.: Sahidic oeile, Bohairic wili 'bélier') is most probably a lw. from Hbr. Rayil 'ram'.

- Cf. SED II No. 24; Mil. 2009; AADB 2466.

\section{3. * $\{a f V w$ - 'k. of lesser bovid'}

Sem. * ${ }^{2} a y p(V) w$ - 'foal, kid': Arab.Safw-, Sifw-, Sufw- 'ânon', Sifw-at-, Sufw-at- 'petit d'âne femelle' II Soq. Seyfif (part. redupl.) 'kid'.

Chad. ${ }^{*} H a f V w$ - 'goat': W. *HVf-Vm-: Ankwe fùm 'he-goat', Tangale amf-o (met.?) 'ram', Tsagu úf-è 'goat' || C. *HafVw-: Hwona wufi-rā 'she-goat', Logone üfu, Kotoko hufu, Mesme afou 'goat'.

Cush. S. * Yafaw- 'goat': Qwadza ufw-anko 'goats' I Ma'a afá 'goat'.

$\Delta$ The Chad. and S. Cush. forms testify to AA ${ }^{*} f$; Sem. ${ }^{*} p$ (> Arab. and MSA $f$ ) may originate from both AA ${ }^{*} p$ and ${ }^{*} f .{ }^{12}$ Cf. Canar.: Gomera tufa (<t-ufa?) 'oveja'.

- Cf. SED II No. 49; Mil. 2009; AADB 2472.

1.4a. * ${ }^{*}(n)_{3}-$ and *2i(n)z- 'k. of smaller bovid'

Sem. * 'aa/inz- and *2Vz- 'she-goat': Akk. (OB on) enz-u (ezz-u, inz-u, azz-atu) 'she-goat, goat (gen. term)' || Ugr., Phoen. $\{z \mid$ Hbr. $\{\bar{z} z \mid$ Aram.: Off. $\{n z$, $\{z$ 'goat', Bibl. \&izz-īn (pl.) 'id.', Syr. \{ezz- 'capra', etc. I Sab. \{nz 'goats' (coll.) | Arab. \{anz(-at)- 'chèvre' || MSA *2ōz: Mhr. wōz, Jib. 2כz 'she-goat', Soq. 2oz 'chèvre, mouton'.

(?) Chad. W. *Haz-an-: Bokkos Zazan 'ram'.

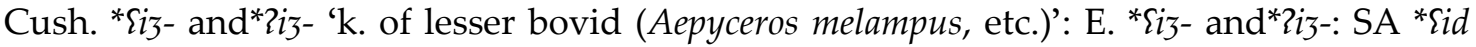
and *idd-: Saho Sid-oo (acc. to Conti Rossini 1913), ayd-ố (acc. to Reinisch 1890), Afar ayd-áa 'sheep' I LEC *2iz-: Somali id-o, Rendille íy(-e) 'flock of sheep', Arbore 2izz-e'gazelle', Elmolo Zéd-i 'goat' || (?) S. *2and-: Asa 2and-o 'impala; female impala' [Aepyceros melampus]. ${ }^{13}$

$\Delta$ Cf. Sum. ùz 'goat, she-goat' (EDS \#2887) and é.ze 'sheep' (EDS \#723).

- Cf. SED II No. 35; AADB 2471.

1.4b. *ma-Si(n)z- 'k. of bovid'

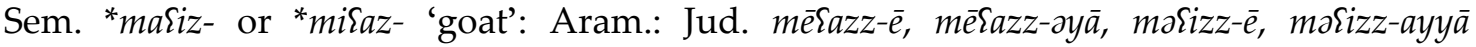
'goats'14 | ESA: Minean $m \mathcal{E z - y}$ (du.) 'chèvre'| Arab. miઈāz-, maSīz- 'chèvre ou bouc'.

(?) Egyp. (Coffin texts) myz-t 'horned animal (?)'15.

elaphus], Hbr. Zayyāl 'fallow deer' [Damadama]; Aram.: Off. Zyl 'deer, stag', etc.; Arab. Ziyyal-, Zuyyal- 'cerf' [C. elaphus].

${ }^{11} \mathrm{Cf}$. Ahaggar élahei 'mouton à laine': met.?

${ }^{12} \mathrm{Cf}$. what is perhaps a variant root with * $p$-: Sem. ESA: Min. fys 'chevreau', Arab. faSfaf-id. (the meaning of both terms is questionable); Egyp. (20 Dyn.) Spwy (met.) 'name of a holy ram' [Ammotragus lervia]; Cush. E.: Dullay:

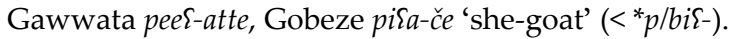

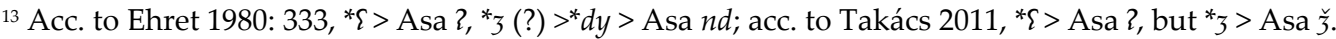

${ }^{14}$ Oddly translated in some Aram. dictionaries as 'from goats, goats-hair, horn, etc.'

${ }^{15}$ Note lack of $-\mathcal{\{}$ - vs. the Sem. forms. 
Chad. *maHa(n)z- 'k. of bovid (Tragelaphus, Kobus); male-goat': W.*maHaz-: Hausa màaz-o 'harnessed antelope' [Tragelaphus scriptus], Mburku maaz-u 'antelope sp.', Bade máz-àrán (compound?) 'Ziegenbock' \|| C.*ma/u(n)z-: Fali-Bwagira munza-ḩwun 'male goat', Logone máz-ā 'Antilopa hamariya' [Kobus kob], ${ }^{16}$ Matakam múz-àk 'he-goat' || E.: Barein múzz-o 'ox' .

(?) Cush.: S.: Dahalo másad-e 'female topi' [Damaliscus lunatus]. ${ }^{17}$

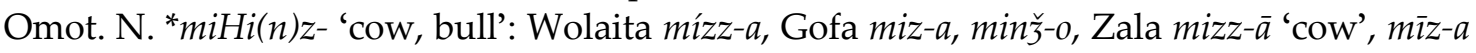
'livestock, cattle', Dorze miiz, Koyra miz-a 'ox', Chara miy-ā, Yemsa miy-ā, miž-à 'cow', Kafa minž-o 'cattle', Bworo mii(n)z-à 'cow, cattle', Dizi (Sheko) mīnž-o or mīnz-a. ${ }^{18}$

$\Delta$ Looks like a unique case of derivation with the prefix $m$ - from * ${ }^{*} i(n)_{3}$ - ' $\mathrm{k}$. of smaller bovid' (1.4a.) as early as the PAA level. With all the problems in each of the proposed roots and/or their relationship, a rather rare combination of the radicals ${ }^{*} \mathcal{E}$ and ${ }^{*} 3$ makes chance coincidence unlikely.

- SED II No. 148; EDE III 156-7; 796; AADB 2695.

1.5. *ba/iz- *barba?- 'k. of lesser bovid (Tragelaphus silvaticus?)'

Sem. ${ }^{*} b i b(b)$ - (redupl.) 'Ovis orientalis, Tragelaphus sp.': Akk. (SB) bibb-u 'wild sheep' [Ovis orientalis] || Tgr. bub-a 'koodoo (antelope)' [Tragelaphus sp.].

Chad. W. *baw? - and *bazb(ar)- (redupl.) 'bushbuck (Tragelaphus sylvaticus)': Sura pàab 'gazelle', Angas bwop 'he-goat', Bolewa ḅoḅ-oki, Karekare bàw-í, Dera bów-ò, Tangale bob-o, Bele bòbòo, Kirfi bòzz-ò, Gera bobb-i, Galambu bùb-à 'duiker' [Tragelaphus sylvaticus].

Cush. S. "bar- and ${ }^{*} b a z b a r-\left(\right.$ redupl.): Iraqw buubuu-ti $\left({ }^{*}-a->-u\right.$ - influenced by $b$ ?) 'gazelle sp. (large and gentle)' I Qwadza bar-uko 'bush duiker' [T. sylvaticus] I Dahalo ḅábą-ááni 'bushbuck' [T. sylvaticus].

SED II No. 54; cf. HSED \#278.

\section{6. *balay/w- 'k. of lesser bovid'}

Sem. *w/yābil- (met.) 'ram': Phoen. ybl, Hbr. yōbēl I Aram.: Dem. Zybl, Jud. yūbalā-'ram' । Arab. wäbil-at- 'petits chameaux ou agneaux; petits d'un troupeau composé de chameaux ou de moutons'. ${ }^{19}$

Egyp. (OK, MK) ib? $2 w$ (very likely < *2VbVl- or * $y V b V l-$, met.) 'Mähnenschaf (Ovis tragelaphus)' [Ammotragus lervia].

Berb. N. *ballVy 'sheep, ram': Gurara, Tuat, Tidikelt belli (pl.) ${ }^{20}$

Chad. *(m-)bil- and *balaw/y- 'small cattle': W. *m-bil-: Geji mbil-a 'sheep' II C. *bVlaw-: Matakam báláw 'race de mouton sp.' ॥ E.*balVy: Lele bulóbuló (redupl.) 'he-goat', Kabalai bâl, Migama bòlíy-ò, Sokoro bal 'goat'.

${ }^{16}$ Cf. also Gudur maygazaw (<*mansaz-?) 'reedbuck' [Redunca sp.], Gisiga mazawal 'male goat'.

${ }^{17} \underline{d}$ is considered to continue only ${ }^{*} d$, but a few other cases of Dahalo $\underline{d}<{ }^{*} /{ }^{*} z$ including the present reflex in the triconsonantal root do not seem haphazard; an alternative comparison can be mō̌̆o (mōdzo) 'sp. large antilope' commented in EDE III 158: "cannot belong here, being a late loan-word from SNil. (as suggested by EEN)”, which in the light of the AA parallels can be, on the contrary, a lw. from Dahalo.

18 Acc. to EDE III 157, «There seems to be a great confusion in the handling of the reflexes of NOm, *miz"Rind"...and NOm. *minž “cattle"... usually mentioned together in Omotic etymologies...» The alternative approach suggested (ibid.): «NOm. *minž “cattle” (palatalized from *ming-?) is fairly close to ES: Amh. mänga, Gaf. mänga 'flock, herd'» is hardly possible as the Omot. languages in question do not show palatalization of the velars $\left({ }^{*} g\right.$ and ${ }^{*} k$ ) while the alleged source Eth. terms show $g-$, not $\breve{z}-$.

${ }^{19}$ Cf. also Akk. būlu 'herd of cattle, sheep or horses; wild animals (gen.)' (unless from Sem. *bawl- 'mind, spirit, living creatures').

${ }^{20}$ Infantile words: acc. to EDE II 24, “therefore cannot be considered” which is a debatable statement. 
Cush. ${ }^{*}(2 a-m-) b V l-~ ' k$. of bovid' (Tragelaphus sylvaticus, etc.): E. ${ }^{*}(2 a m-) b u l-:$ LEC ${ }^{*} b u l a l-$ (redupl.): Oromo bulál-ē 'Lamm' I HEC *2am-bul-: Hadiya ambul-a 'ram', Kambatta ambul-a 'ram, goat' || S. *(2a)m-bal-: Ma'a mbāl-a 'Buschbock' [Tragelaphus sylvaticus].

$\Delta$ Chad. ${ }^{*} m$ - and Cush ${ }^{*}\{a-m$ - are fossilized nominal prefixes or "root-extensions" (see Militarev 2005). ${ }^{21}$

-Cf. HSED No. 2570; SED II No. 245; Mil. 2009; AADB 2484; EDE II 23-24.

\section{7. *baray- *bayar- 'k. of lesser bovid'}

Sem. *bayr-'bull, ox':22 Akk. (OB on) būr-u (pūr-u) 'young calf', (MB on) bīr-u 'bull (for breeding); young cattle (up to three years)' \| Mand. bir-a 'domestic cattle', bar-a 'lamb' I Arab. (Yem.) bār-ah 'cow' | Tgr. bar-a 'ox' I Amh. bare (< *baray) | Har. bār-a 'ox, bull' I Gur. *bawr'ox (for farming)': Cha., Eža, Muh., Msq., Gog., Sod. bor-a, Gye. bawr-a, End. bawr-ä, Enn. bawar-a 'ox (for farming)'.

Egyp. (OK) $b$ ? 'heiliger Bock (Ovis longipes palaeo aegyptiacus)' [Ammotragus lervia] I Copt.: Fayumic barit, Boheiric barhit 'bouc' (- $h$ - is not clear).$^{23}$

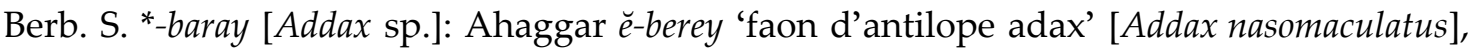
Ayr, E. Wlm. e-bărăy 'faon d'oryx/adax' [Oryx sp./ Addax sp.], W. Wlm. (Kel-Ataram) barwāy-a 'ram'. ${ }^{24}$

Chad. "baray/w- and *babar- (redupl.) 'k. of bovid (Hippotragus, Cephalophus, etc.): W. *baray/w- and *(ba)bar-: Hausa bàrééw-áá, pl. bàrèey-íi 'gazelle', Gera bar-a 'buffalo' [Syncerus caffer], Pa'a bàbár 'roan antelope' [Hippotragus equinus], Siri babar-i 'gazella dama' [Nangerdama], Tsagu bur-āmon 'gazelle', Polchi mbàr-àn 'antelope', Zul bar-námi25 'roan antelope' [H. equinus], 'buffalo' [S. caffer] ॥| C. *bV2/wr-: Masa bo2or-a 'k. of gazelle', Banana bbōr 26 'cephalophe de Grime' [Cephalophus sp.] I| E. *ma-bur-: Lele mbur 'cobe onctueux (Cobe Defassa)' [Defassa waterbuck], Kwang mābár-ā 'duiker' [Tragelaphus sp.].

Cush. *bayr- (met.) 'k. of bovid (Oreotragus, Oryx etc.)':27 LEC *bayr-: Somali bayr 'Antilope oder Oreotragus saltator, Klippspringer' [Oreotragus oreotragus] (Reinisch 1902), báràr (pl.) 'lambs' I| S.*ba?/wr-: Burunge baßur-u 'oryx' [Oryx sp.]. ${ }^{28}$

${ }^{21}$ There are also two phonetically tricky terms: Berb. E.: Ghadames ta-bali, Audjila te- $\underline{b} e l$ 'sheep' which can originate either from *baHl- or from *Hawl- and Chad. C.: Boka ḅwàl-a 'goat' where the voiced implosive is expected to originate from the combination ${ }^{*} b(V) H-$; while no other AA zoonym conforms to such parameters, these forms may be reserved as Berb.-Chad. ${ }^{*} b V H l$-. Cf. similar terms in non-Afras. African languages: C. Sud.: PMoruMadi *bilı 'sheep', Bagirmi bal 'ram'; Ful mbala, mbāl-u, bāli 'sheep'; W. Atlantic: Serer mbal, Mende, Kpele bala id.; Masai em-balelo 'lamb'. Cf. also Chad. W.: *baHil-(Vm-) 'horn': Montol bulu, Bolewa ḅoolum, Galambu ḅàlí, Maha belem, etc.; C. *ti-m-bil-: Bura ti-mbil, Chibak ta-mbalá 'horn' and Omot. S. *bal-: Dime bal-tu, Galila baal-i 'horn'.

${ }^{22}$ Cf. also Proto-Sem. *basir- 'household animal; beast of burden' probably derived from *bayr- with a secondary $-\{$-: Ammonite $b \mathcal{S} r$ 'ox (beast of burden)', Hbr. basir 'livestock, cattle'; Syr. basir- 'brutum, jumentum'; ESA: Sab.b£r 'cattle, head of cattle; camel', Min. $b \mathcal{}$ r 'chameau, tête de gros bétail'; Arab. basìr- 'chameau; toute bête de

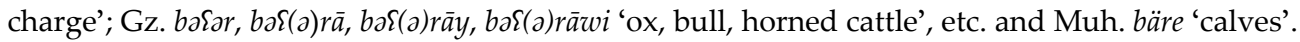

${ }^{23}-r$ - in Fauymic points to ${ }^{*}-r$ - rather than *- $l-$, though, acc. to EDE II, 23, it is "most probably, $<{ }^{*} b l$ ".

${ }^{24}$ Cf. also Ghadames âbbur 'agneau' (Motylinski 1904: 99), not in Lanfry 1973.

${ }^{25}$ nami 'meat'.

${ }^{26}$ It is not clear if $b b$ can convey $b$.

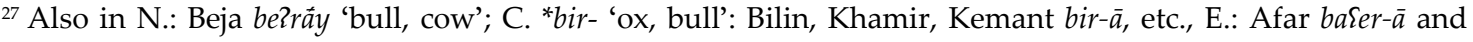
Saho be£er which must all be lws. from N. Eth. *bafray 'bull, ox' (it is not so evident with Afar abur 'bull, ox'), HEC *bāHōr- 'young bull' (Sidamo boor-to, Hadiya baara, Kambatta boora) as well as N. Omot. Wolayta, Zala, Gofu, Kullo, Dache, Zaysse, Chara bōra 'ox', Gamu bōra 'not castrated bull', Kafa bariy-ō 'calf', Mocha bariyo 'steer', Bworo berō, Dizi (Sheko) bariyo 'bull' are more likely lws. from Amh. bare ‘ox, bull'. 
- Cf. AADB 2498, 2487; HSED Nos. 183, 217, 341; ND 225a; EDE II: 24-25, 53 notes, 54-55; Blažek 2003b: 231, 238; SED II No. 53; Mil. 2009; CED \#22.

1.8. *cawiz- 'k. of bovid'

Sem. *'ŝwar- 'sheep': Akk. (OB on) šuนu (š̂̂) 'sheep', (OB) šuātu 'ewe'29 \|I Ugr. š 'ram, sheep' | Phoen $\check{s}$ 'sheep, one of a flock', Hbr. $\hat{s} \bar{a}$ 'small livestock beast'30 | Sab. $s_{2} h$ 'sheep' | Arab. šẫ - 'brebis', šāt- 'brebis, mouton, espèce ovine; buffle (mâle)'.

(?) Egyp. (MK) šw 'ass' (with a meaning shift?). ${ }^{31}$

Chad. *ŝa-32 'cow; meat' (with a meaning shift): W. *ŝa: Hausa šá-nú, šá-nàyee (pl.) 'ox' I Jimi, Polchi ŝáa | Dwot, Buli, Zul, Ngizim ŝáa, etc. 'cow' || C. *̂̃a 'cow': Bura, Margi, Gisiga ŝa, etc. II E. *sVwi: Kera si 'cow', Dormo, Nanchere, Kabalai sī 'ox', Ndam swi 'zebu cow', etc. ${ }^{33}$

Omot. N. *šal- *2ayšs- (met.) 'goat': Bworo ey̌šs-a, Mao (Hozo) šaa, (Ganza) sa?-a, Dizi $\varepsilon s-k u .{ }^{34}$ - Cf. SED II No. 217; Mil. 2009; CED \#831; AADB 229; HSED No. 517.

1.9a. *čąiww- 'k. of lesser bovid'

Sem. *taliw-: Ugr. $\underline{t} 2-a t$ ( $\underline{t} 2-u t)$ 'sheep' I Aram.: Anc. š2-t, siw- $n$, Sam. šs- $h$ 'sheep, ewe', Mand. $t a-t a$ 'lamb, ewe, sheep' | Arab. $\underline{t} a \imath w$-at- 'brebis maigre ou vielle' || Mehri titw-ìt, Hrs. $\underline{t} \bar{i}-t$, Jib. $t \bar{\imath}-t$ 'sheep', Soq. té?-e 'mouton, chèvre'.

Chad. *č $V w$ - and *wVč (met.) 'small cattle':35 W.: Kirfi wǚč $i$ 'sheep' \| E. *č $V w$-: Kwang čuw 'he-goat'. ${ }^{36}$ etc. $^{37}$

(?) Cush. E.: LEC: *čawVZ- (met.) 'meat': Somali so?, Oromo fo-oni, Baiso so?-o, Konso sow-a,

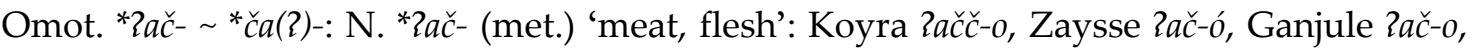
Kachama $2 a c ̧-\jmath$ (the emphatic $\breve{c}$ is notjustified - perhaps a record slip), Chara $a \check{c} \check{c}-a a$, Gimirra $2 a \check{c}$ II S. *ča(2)-: Ongota ča-ta 'meat'.

>Cf. SED II No. 236; Mil. 2009; AADB 2652.

1.9b. *čawir- 'male large bovid or herbivore'

Sem. *tawr- 'Alcelaphus buselaphus; bull, ox': Akk. šūr-u 'bull (poet.)' \|| Ugr. tr 'bull' I Hbr. šōr 'one single beast, bovid' I Aram.: Sam'al šwr-h 'cow', Bibl. tōr 'ox, bull', Syr. tawr- $\bar{a}$ 'bull', Mand. taur-a 'bull, ox' I Sab. $\underline{t} w r$, $\underline{t} r$ 'bull', Qat. $\underline{t} w r$ 'steer, bull', Min. $\underline{t} w r$ 'bull' I Arab. $\underline{t} a w r-$ 'taureau' and 'antelope bubalis' [Alcelaphus buselaphus] (cf. Blažek 2003a: 8) I Gz. sor, ŝor 'ox, bull', sawār 'fat ox' | Tgr. sor 'ox' || Mhr. tawr | Hrs. tawer 'bull'.

${ }^{28}$ Cf. also Cush. E.: HEC *hVbVr-: Qabenna habur-ču 'goat', Burji ibâtr-i 'nanny goat'.

${ }^{29}$ Otherwise $<{ }^{*} \check{c} a r V w-$.

${ }^{30} \mathrm{Jud}$. ŝêtā 'ewe', $\hat{s} \bar{e}$ 'lamb' and Sam. šh 'sheep' are most likely Hebraisms.

${ }^{31}$ Cf. also šr? 'pig, sow', if ? is not $<r$ or $l$.

32 Perhaps ${ }^{*} \hat{s} a$ ?- in view of such forms as ŝáa.

${ }^{33}$ In some of the languages, certain presumably related terms for 'cow' (not quoted here) show a voiced reflex (5), which Stolbova (CED \#831) treats as originating "most probably... from $n t 5<\mathrm{PCh}{ }^{*} n t$ (note $n$ - as a pref. of sg.)" (see in more detail in *ŝa- 'k. of bovid, herd'). Though in CED and other recent works by Stolbova, in accordance with most common usage in present-day Chadic studies, $t$ and $b$ are used instead of $\hat{s}\left(<\mathrm{AA}{ }^{*} \hat{c}\right)$ and $\hat{z}$ $\left(<\mathrm{AA}^{*} \hat{\mathrm{s}}\right)$ accordingly, we continue here to follow the customary latter Afrasian notation. Cf. also the derivative * $\hat{s}(y) V w$ - 'meat' (ibid.)

${ }^{34}$ Omotic reflexes of AA sibilants/sibilant affricates are not quite clear yet.

${ }^{35}$ Cf. W. *čič-: Kariya čiči, Gera čača 'goat', Warji zčizčá-ná, Kariya čícì 'he-goat'. Acc. to Stolbova's observation, only Kariya číci belongs here, while the other forms are $<{ }^{*} a k^{w} i$.

${ }^{36}$ Canarian (all islands) chivato, chiva 'kid' seems more likely < Spanish chibo.

${ }^{37} s$ can continue AA ${ }^{*} s$ - and ${ }^{*} \check{c}$, while Oromo $f$-in foon- $i$, if it belongs here, points to ${ }^{*} c$. 


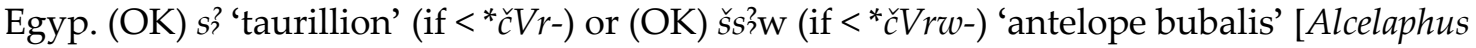
buselaphus]. ${ }^{38}$

Chad. *čiwar- (met.) 'ox; elephant': W.: (?) Dera wór-é 'ox’39 \|| C. *čiwar-: Bura čiwar, Chibak isiwar̃ , Kilba cuwar, Nzangi čuwar-e, Kobochi čūār-é 'elephant' [Loxodonta africana]. ${ }^{40}$

Cush. *čawir- 'bull; hippopotamus, waterbuck': N. *sawsawr- (redupl.): Beja sosar-i 'cattle' \| E.: LEC: Boni šêr 'hippopotamus' [Hippopotamus amphibius] || S. *čawr-: (?) Alagwa cawr-u 'waterbuck' [Kobus ellipsiprymnus] ${ }^{41}$ | Ma'a čur-ú 'bull'.

Omot. N. *šawr-: Kachama šor-o 'rhinoceros' [Dicerasbicornis].

$\Delta$ This root is likely to have originated from \#1.9a. *čaliw- 'k. of lesser bovid' with suffixed ${ }^{*} r^{42}$ and loss of 2 accompanied by an expected deletion of ${ }^{*}-2$ - to size up to a triconsonantal root. The present reconstruction tilts the argument of Sem. ${ }^{*} t a w r-v s$. IE *tauros towards the latter's being borrowed from Sem.

-Cf. SED II No. 241; AADB 2525; Blažek 1994: 200 and Blažek $2003 a$.

1.10. *(2a-)dury- 'k. of bovid'

Sem. *(2a-)dury-: Akk. dudrū 'sorte de mouton' (redupl.) \|| Arab. diry-ān-at- 'espèce de taureau pourvu d'une bosse'। Tgr. (pl.) Zaddari-t 'dwarf-antelope' [Neotragini sp.].

(?) Egyp. (OK) $i d r$ 'Herde (von Vieh und Geflügel)'.

Berb. S. *-dVray- 'oryx' [Oryx sp.]: Ahaggar, Ayr, E.Wlm. e-dari, pl. i-dăra-n.

Chad. *(2an-)dVry- 'Damaliscus sp. etc.; small cattle': W.*(2an-)dur-: Hausa dari 'hartebeest' [Damaliscus lunatus], Guruntum dor-o, dòr-o 'goat', Bokkos 2an-dûr, Sha n-dur 'ram', etc. ॥ C. $\{u-d V r$-: Musgu u-deri II E. *dar-: Dangla dar-o 'gazelle sp.'

Cush. (2a-)dury- 'Tragelaphus sp., etc.': E.: SA *wadar- (met.): Afar wadấr 'Ziegen, Kleinvieh' | LEC * $2 a-d V r y$-: Somali $a$-deri-o 'male kudu antelope' [Tragelaphus sp.] || S. *(da)dury-: Burunge dor-o 'zebra' [Hippotigrus sp.] I Dahalo dádiiiri 'Lesser Kudu’ [Tragelaphus imberbis].

Omot. *dury- 'sheep': N. *dur-: Koyra, Zaise dor-ō, Basketo, Doko dori, Oida dur-o id., Gimira (She) dor 'ram' II S. *dayr- (met.): Ari der-tí 'sheep', Dime der 'goat'.

$\Delta$ Cf. Sum. dàr, dàra 'ibex' [Capra ibex] (EDS \#439).

- AADB 2482. Cf. Takács 2011: 73.

1.11. *fa(2)n- 'k. of large bovid'

Sem. *(w/ya-)pan- 'young bull': Ugr. yp-t 'cow' I Arab. yafan- 'jeune taureau âgé de quatre ans', fan-āt- 'vache' । Gz. ta-yfan 'young bullock', Tgr. tä-fin 'giovenco, bue non ancora domato', Amh. wäyfän (-äy- a diminutive infix?) 'young bullock, calf of 2-3 years', Gur. *wafan 'young bull, steer': Muher, Masqan, Goggot wäfen, Soddo, Wolane m-ofän, etc.

${ }^{38}$ Less likely Pyr. šsr 'Schlachtrind', which may be derived from šsr 'Art des Schlachtens'.

${ }^{39 *} S,{ }^{*} \check{c}>$ Dera $w$, acc. to Stolbova 2009: 28; not in CED.

${ }^{40}$ Cf. C. *či/uw- 'elephant' (unless < Chad. *čVwar- with deleted *-r): Bata čūé, Bana čìwà, Higi-Nkafa čuwe, Mwulien čúwà, etc. C. Chad. (Bura-Margi group) terms like *̌̌VwVr- 'elephant' [Loxodonta africana] are usually considered by Chadicists to be $<* \check{c} V w V n-;$ in Stolbova $2011 \# 433$, the suggested reconstruction *̌̌ $/ \check{c} V w V n$ - is compared with $\mathrm{HEC} * \tilde{3}$ ana? $V y$-; more likely these are two different roots since, acc. to CED: $28-29$, reflexes of $* \check{c}$ and $* \check{z}$ do not overlap, while forms like Bana čìwà are either to be treated as a separate entry (see 1.10a) or point to a deleted *-r rather than * $n$ in word-final position; acc. to CED 35, the former is generally less stable than the latter. At the same time, in CED: $44,{ }^{*}-n>-r$ is postulated for C. Chad. groups 2, 3, 5 and 7, but not for group 4 including NzangiKobochi in which, therefore, $-r<{ }^{*}-r$ is regular which makes the whole idea of ${ }^{*} \check{V} V w V r-<{ }^{*}{ }^{2} V w V n$ - less likely. Cf. also C.: Khamir siriyā 'Antilopensorte'< ${ }^{*}$ čr-?

${ }^{41}$ But Burunge cawadu and Asa šodo id. probably point to *awad-rather than *čawar-.

${ }^{42}$ See the hypothesis on * $r$ presumably conveying the meaning of a "larger dimension/quantity" in Militarev 2005: 49-52. 
Chad. C. *fun- 'buffalo' [Syncerus caffer]: Ga' anda u-fan-a, Higi-Dakwa fun-u, Higi-Kamale n-fun, Fali-Kirya $n$-fu, Banana à-fún-tá, etc.

Cush. S. ' $f a z a n / m$ - 'k. of large herbivore': Qwadza fazamo 43 'buffalo' [S. caffer], Asa fazan-ok 'elephant' [Loxodonta africana]. ${ }^{4}$

$\Delta$ Cf. Bantu *-fuך 'buffalo' [S. caffer].

- SED II No. 250; AADB 2495.

\subsection{2. *(2a-)fir- 'k. of bovid'}

Sem. *parir- (redupl.) and *pa?ur-(met.) 'lamb; young of bovid': Akk. parr-u \| Hatra pr-t? 'ewe or sheep', Syr. parr-ā, Mand. par-a 'lamb' I Arab. furār-, farūr- 'petit de brebisou de chèvre ou de buffle', farìr- 'petit debuffle, de brebis, de chèvre', furfur- (redupl.) 'petit de brebis, agneau ou petit de chèvre, chevreau ou petit d'antilope', (coll.) 'agneaux ou chevreaux', fūr- (pl.) 'chamois' [Capra ibex] II Hbt. fazoór 'male calf (from birth to 5 years)'.

Chad. *(2a-)fir- 'k. of larger bovid' [Tragelaphus, Hippotragus, Syncerus]: W. *fir-: Angas fir 'roan antelope' [Hippotragus equinus] || C. *(2a-)fir-ay-: Hwona $2 u f^{w}$ ar- $\bar{a}$ 'duiker' [Tragelaphus sp.], Bura fir, Kilba fur, Margi fur 'buffalo' [Syncerus caffer], Mbara fàrày 'cattle; dot (bride wealth)'

Cush. E. *2a-filur- 'small cattle': Yaaku apur 'sheep' I| S. *2a-fi/ur- 'he-goat': Asa 2efer-et, 2ofor-ok, Qwadza aful-atu $(-l-<* r)$.

- Cf. HSED No. 1950; SED II No. 181; Mil. 2009; AADB 2494.

\subsection{3a. * guday- 'k. of larger bovid'}

Sem. *gaday- 'kid': Ugr. gdy 'kid'45 | Phoen. gd? 'goat', Hbr. gadī 'kid (of goat or sheep)' ।

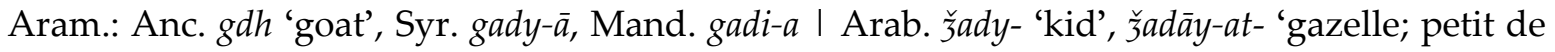
gazelle' [Gazella gazella].

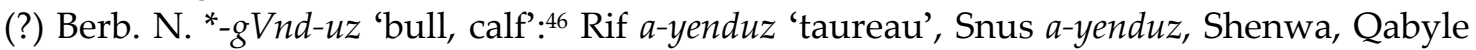
a-genduz, Sened $a$-gendus, etc. 'calf'.

Chad. *ga/uday-: W. *ga/ud-'k. of larger bovid': Hausa gàd-áa 'antelope, duiker sp.' [Tragelaphus sp.], Kariya gud-am, Miya gud-an-zāku, Pa'a gud-an-caka47 'Western kob' [Kobus sp.], Ngizim gád-ùwà 'duiker' [Tragelaphus sp.] \|| C.*gVday-: Zime-Batna gódày 'buck'.

Cush. * gad- and * gund- 'k. of larger bovid': E. * ${ }^{*}$ gad-am-: LEC *gad-am-: Oromo gad-am-sa 'greater kudu' [Tragelaphus strepsiceros], ${ }^{48}$ Dirayta gad-an-sa, gad-am-sa 'antelope' I HEC * gud-: Sidamo god-a 'deer, gazelle' || S. 'gwand-: Iraqw gwand-a, Alagwa gwand-o 'ram', Burunge gond- $i$ 'old ram'.

Omot. N. *gayd- (met.): Zaisse gaaidd-é 'cattle', gaid-é-endo 'buffalo' [Syncerus caffer].

- SED II No. 76; AADB 2490.

1.13b. * ga/ud- 'wild pig'

Chad. *gad-: W.: Hausa gàd-u 'warthog' [Phacochoerus africanus]. ${ }^{49}$

Omot. N. *gud-in-: Sheko gudin-e, Wolaita gudun-ta, Gamu gudun-ci, Daç̌e gudun-ç, Gofa gudun-ça, Kafa gudin-o, Mao kand ( $k-<^{*}{ }^{*}-;$ met.) 'pig'.

${ }^{43}-m<{ }^{*}-n$ assimilated to $f$-?

${ }^{44}$ E. Cush.: Dullay: Harso wayfan-akko, Gollango woyfanna 'bull' are likely loans from Amh.

${ }^{45}$ Akk. (NB) gadî 'male kid' likely < W. Sem.

${ }^{46} \mathrm{It}$ is not clear whether this unusual form could be a metathesis of the compound form * ${ }^{*}$ ed $-n-u z,-n-$ being nota genitivi; for $u z$ see * $\left\{i(n)_{3}\right.$ - and *2i(n)z- 'k. of smaller bovid' 1.4a. (not attested in Berber).

${ }^{47}$ The two latter forms seem to represent word combinations of gud + nota genitivi - $n$ - (see fn. 45).

${ }^{48}$ HEC: Burji gadám-a 'greater kudu' [Tragelaphus strepsiceros] may be a lw. from Oromo.

${ }^{49}$ Cf. Bolewa gedd-o, Mangas gindír, Boghom kindi2, Kiir క̌ìndar 'fat'; Ngizim gád-bàyák 'be fat'. 
$\Delta$ Cf. Kanuri godu, Tubu gadu, Bantu *-gudu 'pig', * gidi 'warthog' [Ph. africanus].

- Cf. AADB 3758.

1.14a. *gawr- 'k. of bovid'

Sem. ${ }^{*}(2 a-)$ gurr-: Akk. (MA) gurr-atu, agurr-atu 'ewe'. ${ }^{50}$

Egyp. (MK) $\underline{d} r$ 'calf' (if $<{ }^{*} g V r$ ).

Berb. *-gur- 'small cattle':51 Ghadames $a$-ร̌ur 'bouc' (Naït-Zerrad 2002: 859), Zenaga a-grarh 'bélier'.

Chad. (a) *(2a-)garw/y- 'kind of bovid (Redunca, Oryx, Ourebia, etc.); animal, game': W. *(2a-)gary-: Hausa āgārēe 'a big, male red-fronted gazelle’ [Eudorcasrufifrons], Tsagu gāre 'reedbuck' [Redunca sp.], Mburku gāri 'oryx' [Oryx sp.], Ngizim a-gare 'gazelle' \|| C. (2a-n-)gary-: Logone gari-a, Makeri ingarii 'antelope', Buduma ygari 'gazelle' II E. *gVrwand *gVwVr- (met.): Tumak garu, Kwang gowor-to 'antelope', Ndam gàrù 'ourebi' [Ourebia ourebi]; (b) *garaw- 'herd of cattle': W. * ${ }^{*} g a r(V) w-$ : Hausa gár-kei 'a herd, flock', Bolewa gaarùw-à 'pack ox', Karekare gaarùw-à 'bull', Tangale káarw-a 'cattle' \|| C. *garaw/y-: Bana gàràw-à 'troupeau de bœufs', Gude gárá-na 'herd of cattle', mà-gárá 'shepherd, watchman', Musgu garí, gári 'Stier, Laststier', Mandara gấri 'bull', Hursa goragor-a(redupl.), Uẑam gwar-a, Matakam y-gwur 'ram' || E. *garaw-: Bidiya gaaruw-o 'animal', Migama gáaráw 'bête,

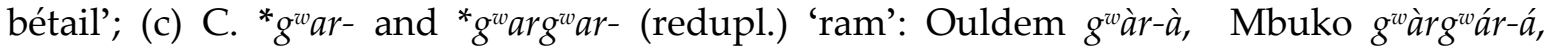
Merey givaràgwàr-a, Muyang g ò̀ròg ò $r-a ̀$, Mada gurgwar-a II E. *gagar- (redupl.) 'small cattle': Sokoro gaáger-o 'Schaf', Mawa gagar 'mouton', Mubi wegr-i (met.) 'chèvre'.

Cush. *garaw- and *gwira?- (met.) 'k. of larger bovid (Taurotragus, Alcelaphus, etc.): N. * garuw-: Beja garuw- $a$ 'male antelope; eland' [Taurotragus sp.] I| C. *gar- 'calf': Bilin, Qwara, Kemant gär, Aungi gara || E.: HEC *gur(r)-: Sidamo gur-um?-iččo 'gazelle', garr-ančo 'Agazen antelope' [T. buxtoni] || S. *gwara?-: Iraqw gwara?-ai 'Hartebeest' [Alcelaphus caama], Burunge gera?-i 'Grant's gazelle' [Nangergranti].

Omot. N. * gaHar-: Wolayta, Dawro gaar-aa 'antelope dekula' [Tragelaphus decula]. ${ }^{52}$

- AADB 2485, 3386, 4152; cf. CED \#234, 235, 238; cf. SED II No. 85.

\subsection{4b. * gur- 'wild pig'}

Chad. E. * gawr- or *gur-: Jegu gor-oך 'Wildschwein', Bidiya gōr-inya 'warthog', gerwa (met.) 'Wildschwein', W. Dangla bó-gór53 'pig doe' (Skinner 1994).

Cush. C. * $g V r-m$ - 'pig': Aungi garm-i, Kunfäl gerim-i (Appleyard 2006: 110).

Omot. N. * gur-it- 'pig': Anfillo gur-ico, Bworo gur-iccà.

- AADB 2683.

1.15. * fawz- 'k. of smaller bovid'

Sem. *'ruzāl- 'gazelle' [Gazella gazella]: Akk. huzāl-u, uzāl-u 'young gazelle' \|| Aram.: Jud.

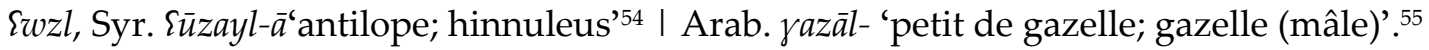

${ }^{50}$ Cf. Sab grh 'domesticated animals' (Biella 1982: 76; not in Beeston et al. 1982), Tna. gahret (-h- is nonetymological) 'doe antelope’. Cf. Gz. garha 'tame, break a horse, mule’. Tgr. gärwa 'kudu (antelope)' [Tragelaphus $s p$.] is likely a lw. from Beja.

${ }^{51}$ Cf. also Ahaggar $\breve{a}-\bar{g} \hat{u} r$, E. Wlm. ă-gur 'animal castré', Adghaq a-žorh 'castrat (caprin)' (Naït-Zerrad 2002: 878).

${ }^{52}$ Lws. from Sidamo?

${ }^{53}$ Acc. to CED \#234, $b$ - is a prefix in E. Chad.

${ }^{54}$ Cf. $\{$ wzll 'porcus'.

${ }^{55}$ Cf. Gz. kazal, Tna. kažal 'kind of antelope' (< Arab., acc. to Leslau 1987: 457) and Chad. C.: Bura kažal 'antilope sp.', Fali-Bwagira kažaran 'gazelle'. A variant root? 


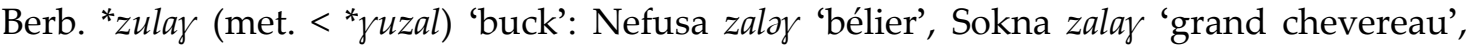
Siwa zalaq, Ghat $a$-žulay, Ahaggar a-hûlay, Ayr a-zolay 'bouc', etc.

Chad. (no single form reconstructable): W.: Hausa ganžar'56 || C.: Gudur maク-gazaw 'Redunca (reedbuck)' [Redunca sp. $]^{57}$

Cush. C. *2a-gaz-in- 'agazen antelope' [Tragelaphus buxtoni]: Qemant agāzēn, Aungi agāzièn-ī.

$\Delta$ Most likely, the root ${ }^{*}$ aww3- was joined with the suffix -al- (later fossilized) at the NAA level, forming Sem. ${ }^{*} \gamma u z \bar{a} l-$, while the P.Berb. form underwent metathesis.

- Cf. AADB 2634; SED II No. 92.

\subsection{6. *hawr- 'k. of larger bovid'}

Sem. *haw(a)r- 'bull, cow': ESA: Sab. hwwr-y, Hdr. hwwr, Min. ḩwr (probably) 'cow' I Arab. hawar- 'taureau' (see SED II No. 106). ${ }^{58}$

Berb. *-Hayr-'calf': Shilh irey (met.) 'veau', Ayr, E. Wlm. e-har-i, pl. i-hăr-an 'jeune bœuf de 2 à 3 ans'.

(?) Chad. *har -: E. *har-: Somrai hār-a 'elephant' [Loxodonta africana]..$^{59}$

Cush. E. *hawr-: LEC *hawr-: Oromo hor-ii 'cattle', Konso hor-eeta 'wealth, livestock'60 । HEC *hur-: Burji húr-i 'buffalo' [Syncerus caffer]. ${ }^{61}$

AADB 4120, 380; cf. Stolbova 2019 \#224.

1.17. * $k^{w}$ ayr- 'young (small) bovid'

Sem. *ka/irr-'small cattle': Akk. (OAkk) kirr-u (or girr-u) 'a breed of sheep' II Ugr. kr 'ram' । Hbr. kar '(young) ram' I Aram.: Off. $k r$ 'sheep' (not quite reliable). ${ }^{62}$

Berb.*karw and *-krar (redupl.) 'young small cattle': Nefusa a-krar'billy-goat', Semlal $i$-kru 'chevreau', án-kkūr, pl. in-ukr-ān'bouc', Rif i-karr-i, Qabyle i-karr-i 'ram', Ghat a-karw-āt 'lamb', Ahaggar ê-krer, pl. a-krâr-en 'bélier', a-kerw-ât, pl. i-kerw-âten 'agneau', etc.

Chad. * $k^{w} a r-$ 'lesser bovid (Redunca, etc.); small cattle': W. ${ }^{*} k^{w} a / i r-$ : Kofyar koor 'castrated goat', Angas kwēr, Dira kur-e 'reedbuck' [Redunca sp.], ${ }^{63}$ Gera kwar-à- 'goat', ${ }^{64}$ Jimbin kwar-iya, Mburku kwaar-i 'meat, animal', Zaar kar-ò 'sheep', Wangday kér-ò 'ram', Kulere kìry-êt 'wild animal; meat' || C. *kwarkwar- (redupl.): Ouldem kwàkwàr 'taureau' || E. *ku/ar-: Kera kúrkí 'taureau', Tobanga (N. Gabri) kar-aך 'goat'.

Cush. E. *kawr- 'goat': LEC: Arbore kaar-íy 'heifer goat', kor-at 'male goat' I Dullay *kawr-: Dobase kor-en-te 'female goat' I Yaaku kúrúm-a 'young goat; lamb'.

- Cf. HSED No.1432; SED II No. 118; Mil. 2009; Stolbova 2011 \#286; AADB 2491.

${ }^{56}$ Diri, Jimbin ganžar can be lws. from Hausa; cf. also Kanuri nganžar which may be the source of the Hausa term or, on the contrary, borrowed from it.

${ }^{57}$ Musgu salak/g 'gazelle' is a lw. from Berb.?

${ }^{58}$ Cf. also Gz. harāwwayā 'pig, wild boar', Tgr. haräwya 'boar', Har. hariyya, Amh. araya 'wild pig', Selti iräyä 'pig', Gur. *haräyä 'porcupine'. Cush. N. *haraw-: Beja harawya; E.: SA *ha(w)r-: Afar horay, Saho hara 'wild boar' seem to be lws. from Eth. or vice versa.

${ }^{59}$ In CED \#361, united with C.: Chibak ḥla 'Stier', Kilba hā-hál-dì 'bull', Musgu halúu, hollú, Mbara hùlúù 'buffalo', where $-l$ - may continue both ${ }^{*} r$ and ${ }^{*} l$.

${ }^{60} \mathrm{Cf}$. Dirayta hor 'multiply (of domestic animals)'.

${ }^{61}$ Omot. N.: Malo hāri, Oyda (h)arr 'cattle' are from Oromo.

${ }^{62} \mathrm{Cf} .{ }^{*} k\left({ }^{\mathrm{w}}\right) a r m-$, probably related, with the suffix $-m$ : Amh. kurm-a 'bull (not castrated)', Har. korm-a 'male (animal), brave', Muh. $k^{w} \ddot{a} r m-a$ 'the young male of a sheep bigger than țäbb ät, male (animal), bull'; cf. also Arab. kawr- 'troupeau nombreux (de chameaux, de bœufs)', 'herd (of camels or gazelles, containing 150-200 animals)'.

${ }^{63}$ Also kìr 'fattened ram'.

${ }^{64}$ Cf. Tangale kârwa 'cattle' and kuri 'herd (of cattle)', kərne 'to protect, guard, keep, shelter, watch', Dera kárán 'herding' < *kVr- 'to guard, to protect, to shelter' in Stolbova 2011 \#259. 
1.18. *lawi2- 'k. of large bovid'65

Sem. *lawiz- and *lawliz-(redupl.) 'k. of large bovid (Bos, Syncerus, Connochaetes, etc.)': Akk. (OB on) litt-u (lìt-u) 'cow', lalû (laliz-u, lalaz-u) 'kid', lulī-mu 'red deer, stag' [Cervus elaphus] ॥ Ebl. lí-a-núm, lí-a-nu-um 'cow' | Ugr. lli-u 'lamb, kid' | Hbr. lèe-a- 'wild cow' [Bos taurus primigenius] (only as a pers. name) I Arab. lä? $a^{n}$ 'taureau sauvage, buffle' [Syncerus caffer], luzluz- 'antilope' I Tgr. lul-it, pl. läwäll-it '(cow) with long, crooked horns', Amh. lil(la)wil 'gnu' [Connochaetes sp.] \|| Mhr. lahay-tan 'cows', Hbt. leé, pl. lháyta, Jib. le?, pl. lhó-ti, Soq. Ze-lh-eh 'cow', lúl-oh 'brébis'. ${ }^{66}$

Egyp. (Pyr.) iw? 'bull' (if <*lVwVz-).

Berb. *w/yalaH- (met.): Izayan ta-wala 'troupeau de bœufs, sangliers', Ahaggar élah-ei 'mouton à laine'. ${ }^{67}$

Chad. *laway- 'k. of larger bovid (Tragelaphus, etc.)': W.: Dera la-à 'cow' \| C.: Gude la 'cow' (Jungraithmayr \& Ibriszimow 1994), Kapsiki, Higi-Nkafa $l \varepsilon$, Higi-Baza $l \supset l-\varepsilon$ (redupl.) 'gazelle', Higi-Ghye le, Bachama líycy 'duiker'[Tragelaphus sp.] (Stolbova 2005 \#57), Masa lúway 'troupeau, le betail' (CED \#549).

Cush. *lawiz-'cattle’: C. *luway-: Bilin luwî́, Khamir luwấ, Awngi luwā \| E. *lar- and *lazlar(redupl.): SA *la(2)-: Saho, Afar lā 'cow, cattle' I LEC *loz(lor)- 'cows (coll.)': Somali lor 'cows (coll.)', Rendille (pl.) loóly-o, Oromo loo-ni 'cows (coll.)', lal-eesa 'she-goat', Konso low-aa 'cows', Dasenech lal-o, pl. lal-i 'cow', etc. I HEC *lal-: Sidamo lal-o 'cows, cattle', laat-to (f.) 'young sheep, lamb' | Dullay *lor-, pl. *le?- 'cow': Harso, Dipina, Gollango lóz-o, Tsamai $l \bar{o} \imath-\bar{o}-$, pl. lēe-e, etc. II S. *liz-: Iraqw le?-i, Gorowa lee?-i 'goat' I Qwadza le?-amuko 'bull'.

AADB 301, 2477. Cf. HSED Nos. 1632, 1647, 2595; SED II Nos. 142, 143; Mil. 2009; EDE I 86.

1.19. *mayn- 'k. of large bovid'

Egyp. (MK) mnmn.t (redupl.) 'herd, cattle'.

Chad. W. *mawan-: Boghom mwò̀ 'ram, sheep' || C.: Uldeme maâ-ŝa 'cow, bull', Musgu mwuy 'buffalo'.

Cush. *mayn-: C.: Qemant miyän 'young cow'; E.: SA: Saho móynoy 'bull'.

Omot. N. *mēn- 'buffalo': Wolaita mien-ta, Male méni, Kachama men-o, Ganjule mēn-o, Gimirra men, Dizi (Sheko) mēn, Mao (Bambeshi) mènè (cf. Kafa mēnō 'meat').

- EDE III 293-4: cf. E. Nilotic: Ongamo (pl.) o-mónyí 'bull', Maasai (pl.) il-móyí 'oxen'; AADB 4261.

1.20a. *mar(ay)- 'k. of large bovid'

Sem. (a) *marVy- 'bull': Akk. (SB) mīr-u 'young bull', (OB) mīr-tu 'cow'll Hbr. marì̄(2) 'fatted steer'68 | Arab. māriyy-at- 'antilope blanche'.69

Egyp. (Pyr.) $m_{2}$ 'oryx-antilope' [Oryx sp.] (if $\left.<{ }^{*} m V r\right) .{ }^{70}$

Chad. *ma/uray- 'Kobus sp.': W. *maray: Hausa mààrír-ì 'white oryx' [Oryx leucoryx], ${ }^{71}$ máárày-áá 'Western kob (medium antilope)' [Kobus kob], Saya mááriy-á 'antelope', Warji mārai, Ngizim máarày-á 'kob' [Kobus sp.] || C. *mur-: Masa murí 'antilope sp., prob. kobdéfassa' [K. ellipsiprymnus] ॥| E.: Toram múr-o 'gazelle'.

${ }^{65}$ Cf. Chad. * $l V w(h)$ - 'wild animal(s), meat' (CED \#549), likely related.

${ }^{66}$ Note non-etymological $-h$ - in MSA.

${ }^{67}$ Cf. also alu 'bull', quoted in EDE I 86 as “Tamasheq”, dialect name and source not specified.

${ }^{68}$ Usually regarded as derived from the verbal root * $m r$ ' 'to be fat', which is less likely in view of the Akk. parallel.

${ }^{69}$ Unless 'white, milk-colored' is Arab. miry-at- 'lait qui sort en filet abonant du pis de la femelle' (BK II 1097-8).

${ }^{70}$ Cf. also (late) $m r$.t (if < * $m V r$-, not * $m V l$-) 'cow'; cf. also (MK) $m r$, $m r y$ 'Kampftier' with the sign for bull/cow'.

${ }^{71}$ Less likely < Arab. māriyy-at- 'antilope blanche' in view of other Chad. data; however, the similarity is somewhat striking. 
Cush. E. *mar-: LEC * mar-: Rendille máar 'male calf', maár 'female calf', Arbore máar 'calves' (coll.) | HEC *mar-: Hadiya moor-a 'older calf' I Dullay *mar- 'heifer': Harso, Dihina maar-akkó, Tsamai mār-e || S. *ma(w)r-: Ma'a mor-o 'ox, steer'72 | Dahalo morr-o 'duiker' [Tragelaphus sp.].

Omot. N. *mar-: Gamu mar-a, Dorze mar, Male marr-o 'calf' (probably lws. from E. Cush.)

- SED II No. 153; AADB 2331, 2460; EDE III 26, 393-4; HSED 1728; Mil. 2009.

1.20b. *(2a-)mayr- 'k. of small bovid'

Sem. *2a/i-mmar- 'lamb, sheep': Akk. (OA, OB on) immer-u 'sheep; sheep and goats; ram', (OB on) immer-tu 'ewe, sheep (as a generic term)' I Ugr. Zimr (and 2amr) 'ram' । Phoen. 2mr, Hbr. Zimmēer I Aram.: Anc., Off. $2 m r$ 'lamb, sheep', Bibl. 2immēr 'lamb', Sam. $2 m r$, Syr. 2emr-ā 'lamb', Mand. Sa/umbr-a 'sheep, lamb' I Arab. 2a/immar- 'lamb'.

Chad. W. *(2a-)mar- 'ram, goat': Tangale mar-a '(castrated) goat', Dirì mar-i 'goat', Bokkos maray, Sha àmar-á, Fyer Zamar-a 'ram'. ${ }^{73}$

Cush. E.: SA *mar-: Saho, Afar mar-̄̄ 'ram'.

Omot. *mar(ay)-: N.: Wolayta mār- $\bar{a}$ 'calf, lamb, kid', Gamu mar-a, Dorze mar, Male marr-o 'calf', màrày-i 'ram', marmar-o (redupl.) 'lamb', ${ }^{74}$ Koyra mar-a 'ram', Bworo merēr-à- 'sheep', etc. ${ }^{75}$

- SED II No. 5; AADB 2591; EDE III 42, 394; HSED 1729; Mil. 2009.

1.21. *nąiw- 'k. of bovid'

(?) Egyp. (NK) wnwy 'Kalb (als Bezeichnung des Osiris)'.

Chad *naHaw-: (a) W. *na 'cow': Tangale ta-na | Boghom, Tala, Jimi naa \| C. *naw: Lame náò I Mesme nau, etc. 'cow' I Mandara náw-è 'goat'. ${ }^{76}$

Cush. *nåiw-: N.: Beja naa? 'sheep' || C. *naw- 'calf': Khamta, Khamir niw | Kailiña nāw | Aungi nēw, Kunfal nāww-i $i^{77} \|$ LEC * $n V w-$ : Boni núi 'greater kudu' [Tragelaphus strepsiceros].

- AADB 2025, 2530; HSED, 42; Blažek 2003b: 234; Stolbova 2005 \#364, 364a; Mil. 2009; CED \#585 (comp. with Hbr. and Arab.).

1.22. *saraw/y- 'k. of bovid'

Egyp. (OK) s? (if < ${ }^{*} s V r-$ ) 'Herde'.

Berb. S. *-saraw/y- 'troop, herd': Ahaggar ă-sära, pl. i-särâ-tän 'troupe d'animals sauvages', Taitoq a-sera 'troupeau de moutons, de chèvres, d'ânes', Tadghaq ta-säraw- $t$ 'small herd of cows (up to about 30)', ta-säray-t 'large herd (esp. of camels or cattle)'.

Chad. W. *sa(r)sar- (redupl.): Hausa sassar-o 'male red-fronted gazelle' [Eudorcas rufifrons].

Cush. E. *sar(a)w/y- and *sawr-: SA *saraw-: Saho saraaw-ii 'einzweijähriger zur Arbeit noch nicht abgerichteter Stier' I LEC *sary- 'Oryx, Madoqua': Somali sary-an 'adult male oryx' [Oryx sp.] I Dullay *sawr- 'antelope dikdik’ [Madoqua sp.]: Gollango sawr-ó, sayr-ó, Tsamai sawr-o. ${ }^{78}$

AADB 299. Cf. Blažek 2003a: 9.

${ }^{72}$ Acc. to Ehret 1980, < NS, but in light of other Cush. and AA parallels this seems less likely.

${ }^{73}$ And, perhaps, Tala màar, Buli maro, Polchi mar 'goat' unless < *mad-. Cf. also W.: Dwot maaríiri 'rhinoceros'; C. *maray 'bull': Mofu-Gudur maray 'taureau engraissé à l'étable', Matakam mari 'bull', Mafa maray 'fête rituelle de taureau; taureau de case sacrifié lors la fête'.

${ }^{74}$ Diminutive reduplication?

${ }^{75}$ Ongota mar-te 'she-calf' < Tsamai.

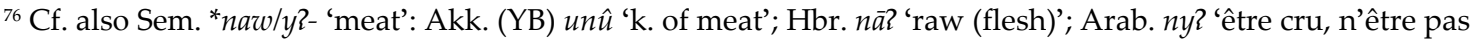

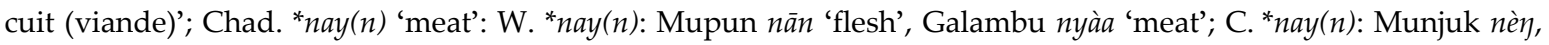

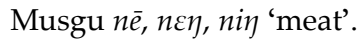

77 Tgr. naway 'cow, possession/fortune' is most likely from Agaw.

${ }^{78}$ Omot. S.: Ongota saire 'antelope dikdik' [Madoqua sp.] is from Tsamai. 


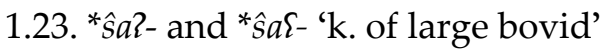

Berb.: Ahaggar êsu (< ${ }^{*} H i \hat{s} w$, met.) 'bull'. ${ }^{79}$

Chad. *zâH- 'large cattle, cow': W. *zâHa: Siri żáà-ti, Dott zááa, Buli, Dott záa 'cow', etc. \| C. ${ }^{*} \hat{z} a$ : Tera $\hat{z} a$, Gude $\hat{z} a ̀ a$, Buwal $\hat{z} a ̀$, Sukun $\hat{z} \jmath .^{80}$

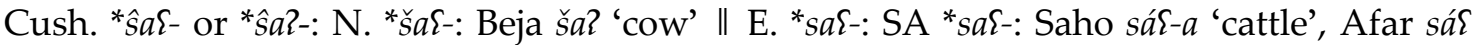
'small stock (animal)' I LEC *saf- 'cow': Somali saీ, Jidda sii, Boni sáz-a, Rendille sah, Oromo sáz-a, Konso sa-a, Bayso se, Arbore, Dasenech se? I HEC *saz-: Sidamo saz-aa, Darasa sa-aya, Kambatta saz-a, Hadiya saa-yya, Burji saa | S. * $\hat{s} a\}-V y$ - 'cow': Iraqw, Alagwa, Burunge $\hat{s} e-e^{81}$ । Asa ŝe-ok I Qwadza ŝae-ko.

-Cf. HSED \#517; AADB 381; CED \#831; Mil. 2009.

1.24. *wasil- 'k. of lesser bovid'

Sem. *waSil- 'mountain goat (Capra ibex, Arabitragus jayakari): Ugr. ysl 'mountain goat' I Hbr.

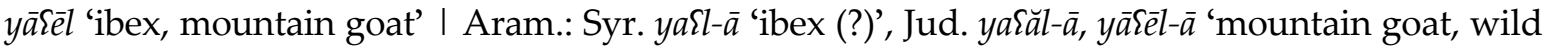
goat' [Capra ibex] I ESA: Sab., Qat. wsl 'mountain goat, ibex' [Arabitragus jayakari], Hdr. 2-wsl (pl.) id. I Arab. wasl-, wasil-, wusil- 'argali, bouc de montagnes' [Ovis ammon]; bouc domestique' । Gz. $w a\{a l-\bar{a}, w a \hat{a} l-\bar{a}, w \bar{a} z a l-\bar{a}$ 'kind of antelope, mountain goat' [C. ibex], Tgr. waSal-a 'elk/élan' (DRS 578) [Cervus elaphus], Amh. waliy-a 'Walia ibex' [C. ibex] II Mhr. wēl, pl. wasōl, waysōl, Hrs. wāl, Jib. $\varepsilon b\{\underline{z} \hat{z}$ 'Hemitragus Jaykari, mountain goat' [A. jayakari], Hbt. wraál, pl. woryoól 'wild goat, oryx'.

Berb. *wulli 'small cattle': Qabyle ulli 'sheep', Ghadames welli, Ayr, E. Wlm. walli, Ahaggar, Tadghaq ulli 'goat'.

Chad. *wayl- 'k. of lesser bovid': W. *wal-: Jimi wal-aróo 'antelope', Geji wull-i 'gazelle' \|| E. *wil-: Lele òl, Kabalai yil-a 'antelope'.

Cush. E. *wisl- * *awl- (met.) 'k. of lesser bovid': LEC * ¿awl-: Somali Eawl-kii, Rendille hol $\left(h<{ }^{*}\right)$ 'gazelle' || HEC *will-: Sidamo wil-ii'l-icco, pl. wil-ii'le 'young (of sheep, goat)'.

- SED II No. 244; AADB 2470.

1.25. *wad- 'k. of lesser bovid's2

Sem. *wad-: Arab. wad-ān- 'mouflon' [Ovis orientalis].

Berb. *a-wdad (part. redupl.) 'Barbary sheep (Ammotragus lervia)': Nefusa a-wdād, Semlal, Izdeg, Ghadames, Ghat udad, Ahaggar ūdad.

Chad. *wad- *daw- (met.) 'k. of lesser bovid': W. *wad-: Hausa ud-a 'k. of sheep' II C. *daw/y: Mofu dáw 'goat', Zime-Batna dèy 'duiker' [Tragelaphus sp.].

Cush. E. *2id-'sheep': SA *Zayd- 'sheep': Saho ayd-ố 'sheep (coll.)', Afar id-a 'ewe' I LEC *2idad- (redupl.): Bayso idādo 'sheep'. ${ }^{83}$

${ }^{79}$ Perhaps also P. Berb. *isa- $n$ (pl.) 'meat' which may alternatively originate from AA * $s V$ z- 'meat'.

${ }^{80}$ In CED \#831 (where $\hat{z}$ is rendered as $\not 5$ ), the above forms are united with the forms in $\hat{s}$ - (rendered by $t$ ) with the following comment: "The word for 'cow' shows irregular voiced reflex $\left(b_{5}\right)$ in a number of Ch Biu-Mandara languages. Most probably, 5 - originates from $n 5<\mathrm{PCh}^{*} n$ - (note $n$ - as a pref. of sing.); this ingenious explanation "reconciling" the reflexes of two different AA laterals (Chad. $\hat{s} / t<\mathrm{AA} * \hat{c}$ and Chad. $\hat{z} / \bar{\zeta}<\mathrm{AA} * \hat{\mathrm{s}}$ ) is opposed by an alternative solution proposed herein.

$81 ?$ is $<* 2$, not $<*$ in $W$. Rift languages.

${ }^{82}$ Cf. also miscellaneous terms that are somewhat similar and probably eventually related: Sem.: Akk. (lex.) $d \bar{u} d u$ (<*dawd-, partial reduplication?), (OA, SB) aletūdu 'wild sheep (male)'; Off. Aram. (hapax in Frah) $2 n d w t\left({ }^{*} 2 V-\right.$ $n$-daw-t?) id. (cf. SED II No. 44) and Cush. *2ad(d)Vy- (?): E. *2addi 'large cattle': SA.: Afar addi 'young heifer', HEC: Hadiya addi-cco, Sidamo adde, Burji addi 'heifer'; S. *de?- (met.?); S.: Qwadza de?- 'to herd', Ma'a dé 'cow, cattle'. Cf. Blažek 2003b: 244 (Beja da 'newly born calf' and the E. and S. Cush. forms are probably not related, since Beja $d$ does not continue ${ }^{*} d$ ).

${ }^{83}$ Difference in the first radicals and stem patterns makes cognation with the present root questionable. 
$\Delta$ Cf. Sum. udu 'sheep' (EDS \#2678).

- AADB 2531.

1.26. *(2a-)w/yan- 'k. of lesser bovid'

Chad. (2a-)yan-'goat': W. *yan-: Siri yáàn-í \|| E. *Zayn- 'goat': Birgit zàyn-éy, Migama ĩ:n-á, Jegu Ràyn-éy (té-én-ó, pl. Réén 'he-goat'). ${ }^{84}$

Cush. ${ }^{*}(2 a-) w / y a n-:$ N.: *2an-: Beja an-o 'sheep' II LEC *wan-: Somali wan, pl. wanan 'ram', Rendille onó 'sheep' । HEC *2ayn-: Burji ayáan-e 'gazelle'. ${ }^{85}$

Omot. S. *yVn- 'sheep': Dime iin, (?) Ongota hoona (h- probably renders ?).

- AADB 2530.

1.27. *war- 'k. of larger bovid'

Egyp. (MK) wr.t 'göttliche Kuh', wr 'Steer', in dem Ortsnamen.

Berb. * $w V r w-(<* 2 a r w)$ : Semlal ta-uru-t 'troupeau (de bœufs)', Azilal ta-wru-tt 'troupeau'.

Chad. W. *war-: Hausa wariyy-a 'a k. of antelope', ${ }^{86}$ Dera wor-e, Kariya wúr-ù, Warji ruwe (met.) 'cow'.

Cush. E.: HEC *war-: Sidamo war-aamo 'older calf'.

- HSED 2527; AADB 2497.

\section{Camels and equids}

\section{1. dawk- 'k. of equid'}

Sem. *kawd-an- (met.) 'mule': Akk. (OAkk. on) kūdanu (kūdannu) ॥ Ebl. gú-da-núm «kūdanum> | Ugr. kdn-t | Aram.: Off. kwdn, Palm. kwdn, Syr. kūdan-yā, kūdon-ā | Arab. kawdan-, kawdan-iyy-. ${ }^{87}$

Chad.: W. *daw(a)k- 'horse': Hausa dốk-i, Karekare dókú, Miya duwak-ə, Burma dok, Ngizim dùk-à, etc. \| C. ${ }^{*}(n-) d o k$ : Tera doh 'stallion', Piẑimdi-Hina n-dok 'pony' \|| E. *dawk-: Dangla dewk-i 'antilope-cheval' [Hippotragus equinus].

Cush. S. *dak-et-: Iraqw dak-eti, Alagwa dek-eti 'zebra' [Hippotigris sp.]. ${ }^{88}$

-SED II No. 124; AADB 2639; cf. HSED 667.

\section{2. * gayl- 'camel'}

Sem. (a) *2a-gayl- and *gilgal- (redupl.) 'k. of equid': Akk. (OAkk. on) agāl-u, agall-u 'an equid' (CAD a1 141), 'Reitesel' (AHw: 15) \| Ebl. ag-lum = ANSE.NITA.KUR (a donkey) । Tgr. galgäl 'foal (mule, horse)', Tna galgal 'puledro di cavallo o mulo non ancora domato' (Bassano 1918: 815), Amh. galgäl 'the young of domestic animals (goat, sheep, horse, donkey)'; (b) * gimal- (met. < ${ }^{*}$ gayl-am-) ${ }^{89}$ 'camel': Hbr. gāmāl । Aram.: Off., Palm., Nab. gml, Syr. gaml-ā | Sab. gml | Arab. ̌̌aml-, ̌̌amal- | Gz. gamal || Jib. gũl (pl. gmíhl), Soq. gimál.

${ }^{84}$ Cf. Can. ${ }^{*} H a n(y)-:$ Tenerife ana, haña, jana 'sheep'.

${ }^{85}$ Cf. also Afar anaీ-to 'lamb (female)' (non-etymological - ). Sem. Gur.: Cha. onā, Eža onnā 'young male goat or sheep' must be from Cush.

${ }^{86}$ Cf. also warari 'a vicious bull' (Skinner 1996: 283).

${ }^{87}$ Likely related is Tgr. mäkada, name of the small Abyssinian horse.

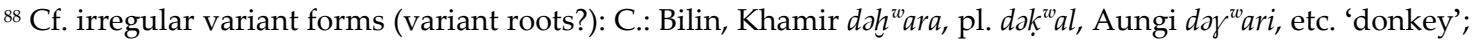
E.: LEC: Oromo donkoro 'stupid, fool', (Bararetta dial.) dongorra 'donkey'; S.: Iraqw, Gorowa, Alagwa, Burunge dakwai 'donkey', Qwadza dagwagwai-ko (Dolgopolskiy 1973: 275), dak ${ }^{w} a k^{w} a y-i k o$ (Ehret 1980: 345). Cf. also Chad. C. ${ }^{*} z V n g^{\mathrm{w}}$ - ‘donkey' (Stolbova 2009 \#530).

${ }^{89} \mathrm{Cf}$. a similar process in *himār $-<$ *hayr-ām-(2.4). 
Berb. * gilgal- (redupl.): Ahaggar $\breve{a}$-gelgâl- $i$ 'chameau de charge commun et lourd'. ${ }^{90}$

Cush. E. * gayl- 'camel': SA *gāl-: Saho, Afar gál-ā \|I LEC *gayl-: Somali geel-a (coll.), Boni g'áál, Rendille gaal, Oromo, Baiso gaal-a, Arbore gaal, Elmolo gal-te. ${ }^{91}$

AADB 2508; cf. SED II No. 79.

\section{3. *hilul(l)- 'k. of equid'}

Sem. *hill(l)-ay- 'colt, foal': Akk. (OB) ilulay-u 'ein Kamel?' II Tgr. hel-e 'camel (poet.)', Tna. haw l-a 'donkey's colt or foal of about two years' II Hrs. me-heley-ōt 'she-camel with fully-grown young'.

Berb. S. *hulil (redupl.) 'wild ass, foal': Ghat a-hulil 'âne sauvage' [Equus asinus], Ahaggar $\breve{a}$-hâhul 'jeune chameau non castré', Ayr, E. Wlm. a-hulel, pl. i-hule-tăn 'poulain, ânon'.

Cush. E. *2i-ḩVll- (redupl.) 'k. of equid': LEC*(2i-)haw/yl-: Rendille é-hel, Arbore holl I Dullay *(H)all-: Gollango ooll-ó 'Pferd' \|| S. *ḥVllVz- (met.?): Dahalo ḥellez-a 'zebra' [Hippotigris sp.]. ${ }^{92}$ AADB 2462.

\section{4. *ha/ir- 'wild ass'}

Sem. (a) *hayur- 'ass, camel': Arab. huwwār-, hivwār- 'petit de chameau récemment né, ou jusqu'à l'époque du sevrage'93 || MSA *hayr-, pl. hayor:94 Mhr. hayr'male donkey', hīr-ìt 'female donkey', Hrs. hayr 'donkey', hayr-ēt 'she-donkey', Hbt háyr, pl. hyeér (m.), hiiirí-it, pl. hiyór-ta (f.) 'donkey, ass', Jib. (Kathīri dial.) a-hyyćr 'male donkey'; (b) *himār - 'wild ass, onager, donkey' (met. < *hayr-ām-): ${ }^{95}$ Akk. (OAkk. on) imēr-u 'donkey, male donkey' II Ugr. ḩmr; Hbr. ḥ̆mōor 'he-ass' I

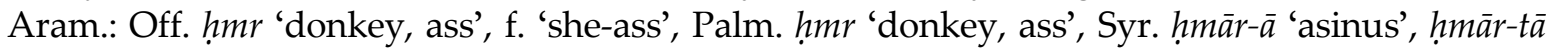
'asina', Mand. hamar-a 'pack-animal, ass, donkey' I Sab., Min. hmrr 'ass; wild ass, onager' [Equus asinus, E. hemionus], 'domesticated ass' I Arab. himār- 'âne; âne sauvage, onagre' [E. asinus, E. hemionus], himārat- 'ânesse' ${ }^{96}$ | Gur. *amar 'donkey': Cha. Gog. amar, Sod. ämar, Wol. umar, etc. ${ }^{97}$

Cush.: N. *har-: Beja har-e 'camel' (Blažek 2003b:13)98 || E. *ha(y)r- 'wild ass, donkey': SA *hayr-: Saho her-a 'female donkey' I LEC *harr-: Oromo harr-ee, Konso harr-eta, Dirayta harr-et 'donkey', Dullay *har(r)-: Gawwada, Dobase, Golango harre, Harso har-iččé 'ass' [Equus asinus]. ${ }^{99}$

- SED II Nos. 98, 106; AADB 2463, 4120.

\section{5. ${ }^{*} k^{w}$ ar- 'wild ass or camel'100}

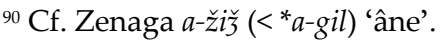

${ }^{91} \mathrm{HEC}{ }^{*} g \bar{a} l-:$ Sidamo, Darasa gaala, Burji gaali; Dullay ${ }^{*} k \bar{a} l-\left(<{ }^{*} g \bar{a} l-\right)$ : Gawwada kaal-hó (< $\left.{ }^{*} k \bar{a} l-k-\right)$, Harso, Dihina, Gollango kaalá probably from Oromo. Omot.: Dache, Zaysse gaale, Yemsa gaala, Kafa gallo, Dizi (Sheko) gaale 'camel' perhaps also from Oromo, probably via HEC.

${ }_{92}^{2}$ Omot. N.: Zaisse ollō, wollō, Ganjule ollo 'horse' are probably lws. from Dullay.

93 Tgr. howar 'young (of camel or donkey)' is likely an Arabic loan. Berb.: Ahaggar awra, pl. iwerân 'chameau dans sa 1ère année', Ayr, E. Wlm. awăra, pl. iwăran 'chamelon de quelque mois' are probably Arabisms.

${ }^{94}$ Erroneously related to Sem. * aayr- 'donkey' in SED II No. 50, analyzed as forms with the definite article $h$-. However, this article occurs only in "words with an initial radical 2... and certain...monosyllables with an initial w or y radical" (Johnstone 1987: 163-4). There seems to be no cases of the $h$-article replacing initial etymological $\varepsilon$-in either Mhr. or Jib.

${ }_{95} \mathrm{Cf}$. a similar process in * gimal- $\left(<*^{*}\right.$ gayl-am- (2.2).

${ }^{96} \mathrm{Cf}$. also ahmmūr-, yahmmūr 'onagre' [Equus hemionus].

${ }^{97}$ Cf. Mhr. h̆omūr 'to tame, break (a horse)', Hrs. ḥemōr 'to tame, break (a riding beast)', Jib. hõ r 'to tame, break, domesticate (an animal)'.

${ }^{98}$ Cf. harri 'mount (anything that can be ridden)'.

${ }_{99}$ Wolamo hariya, Gofa haare, Gamo, Dorze, Cancha, Kullo hare, Malo, Dache, Zayse hare, Kachama haarre, Ari harra 'donkey' - likely lws. from Oromo.

${ }^{100}$ Cf. NAA *kawr- 'load, carry a load': Sem.: (a) Arab. kwr 'porter qqch. sur son dos', II 'empaqueter (des bagages)' (BK II942), (b) 'pack saddle, bat': Hbr. kar 'bât (de chameau)', Arab. kūr- 'selle de chameau', Tna, Tgr. kor, 
Sem. ${ }^{*} k^{w} V r r$ - and ${ }^{*} k^{w} V r k^{w} V r$ - (redupl.) 'she-camel': Hbr. kirkär-ät 'she-camel' (redupl.) I Arab. kurr- 'ânon, poulain' (DRS: 1283) ${ }^{101} \mid$ Amh. $k^{w} \partial r k^{w} \partial r$, Wolane kurkur 'interjection one uses when calling a donkey' (redupl.) \| Hobyot kóokar (redupl.) 'female camel (from 2 to 3 years)' ${ }^{102}$

Berb. S. *-kirkar (redupl.): Ayr, E. Wlm. i-karkar (pl.) 'camels'.

Chad. *kwar-: W. *kwar- 'wild ass, donkey': Chip kor-o, Bolewa kor-o, Ngizim kwár-á, etc. \| C. *kwar- 'ass': Tera kór-ó, Hwona, Margi, Gude kwar-a, Banana kwar-a103 \|| E. *kur- 'ass': Ndam kùr-ō, Kwang kúr-ā, etc.

Cush. *ku/ar-: N. *kar-: Beja kár-ee-see 'Kamellaus' (see 'louse’) \|| E.: LEC *(2Vn-)kur-: Somali kór-on 'castrated camel; barren she-camel', Rendille in-kuraarr-ú 'donkey colt, young donkey'.

Omot. N. *kur- 'donkey’: Chara kur-ā, Kafa, Mocha, Anfillo kur-ō, Gimirra, Dizi (Sheko) kur.

-AADB 2468.

\section{6. * piral- 'k. of equid'104}

Sem. * para?- 'onager, wild ass' [Equus asinus, E. hemionus]: Akk. (OB on) parû 'Onager, Maultier' || Hbr. pärä(2) | Sab. fr? | Arab. fara?-.

(?) Egyp. (18 Dyn.) rnp (met. < $p r-n$ ?) 'young horse'.

Chad. W. *pirr- 'k. of equid': Gerka pirr-i 'mare' \|I C. *pur-: Bura pur-u 'white horse'. ${ }^{105}$

Cush. *filar- 'k. of equid': $N{ }^{*}$ firr-: Beja firr-at 'she-camel just foaled' \|| E. *far-: SA *farar(redupl.): Saho, Afar farar 'horse' । LEC *faraw: Somali, Arbore faraw 'zebra' [Hippotigris sp.].106

$\Delta$ Seems like a genuine Proto-Afrasian term for a kind of equid.

- SED II No. 176; AADB 2503.

\section{Largest herbivores ${ }^{107}$}

\section{1. *da(n) $g^{w}(V r)$-'elephant' [L. africana]}

Chad. E. *dugur-: Gabri dūgūur-ùu, Sokoro dogol $\left(-l<{ }^{*}-r\right.$ ?). ${ }^{108}$

Amh. kor-əča, Arg. kor-əčča 'selle' (DRS 1202), Amh. kārkar 'louage des bêtes de somme' (DRS 1276); Chad. (a) *kVr-, kVrkVr- 'load, carry a load' (CED \#446): W.: Bolewa, Karekare karài 'load', Ngizim kákárâ 'load', kàkàrú 'carry a load', Bade karày 'goods', Duwai kàrkàrà 'load carried on the head', C.: Bura kari 'goods, possessions', Fali-Kirya $k^{w}$ àr 'to unload', E.: Tumak gàr 'carry; a load' ( $g$ - <*k- is regular), (b) W.: Hausa ta-karkari 'pack ox'.

${ }^{101}$ Cf. kawr- 'troupeau nombreux (de chameaux, de bœufs)'.

102 Note a striking similarity both in form (reduplication) and meaning (she-camel) in Hbr. and Hbt. Cf. also Amh. kärkar 'louage des bêtes de somme'.

${ }^{103}$ Cf. Nzangi kara, Bata-Zumo kara 'horse'.

104 There are several (five, to be exact) somewhat similar triconsonantal roots in which it is not easy to distinguish between chance similarity and borrowing from suppletive stems such as Somali faras and pl. fárdó, Bilin fardâ and pl. fáriž, bädrā and pl. bäzir, Khamir bázrā and pl. baris. Two of them are well represented in Sem. ("pirdand *paraš-), nearly all in E. Cush and all in C. Cush while a few Chad. and Omot. parallels are most likely loanwords; notably, they seem to have no parallels in Beja, S. Cush., Egyp. and Berb. Surprisingly, all of them mean 'horse', which apparently excludes their PAA status. This entangled situation is worth a separate piece of research, which the authors are planning to publish soon.

${ }^{105}$ Hardly named after its color, since there seems to be no corresponding color name in the available Bura lexicon.

${ }^{106}$ Omot. N. *far- 'horse': Wolayta, Zala, Dawro, Gofa, Gamu, Chara far- $a<$ SA?

107 Some examples from individual languages are quoted after Blažek 1994; however, distribution of the forms among different entries, based on regular consonantal correspondences, is in most cases quite different from this highly valuable collection of data.

${ }^{108}$ Also dúger or dúkur 'rhinoceros'. Cf. Dangla dugulo, Migama divillu 'trunk' and W. Saharan: Daza dugugul 'trunk of elephant'. 


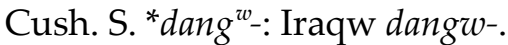

Omot. *dang-Vr-: N.: Zaise dongor, Kafa dangiy-ō || S.: Ari dangór, Hamar donger.

- AADB 2519. Cf. Blažek 1994: 199.

\section{2. *duhr- 'elephant'}

Egyp. (late) $d n h r$ (met. $<* d h r-n$ ?)'elephant' [Loxodonta africana].

Chad. W.*dor-in-'hippopotamus' [Hippopotamus amphibius]: Hausa dò̀īn-a, Mupun dòrin-a, Miya dòrin-á, Tsagu dòrán-à (the last three words can be lws. from Hausa).

Omot. *duHur- 'elephant' [L. africana]: N.: Dizi (Nao, Maji) dōr, (Sheko) door II S.: Dime duur-o.

- AADB 2524; cf. Blažek 1994: 199.

\section{3. *fil- 'a proboscidean mammal'}

Sem. * pìl- and *pVlpVl- (redupl.): Akk. (OB) pìl-u \| pB. Hbr. pìl । Aram.: Syr. pìl-ā, Mnd. pil(-a) | Arab. fil- 'elephant' [Loxodonta africana] I Gz. falfal 'water buffalo; elephant' [Syncerus caffer; L. africana].

Chad. *pil-: C. *pil-: Bura pili-ngir109 'aardvark' [Orycteropus afer]. ${ }^{110}$

Cush. S. *fil-: Iraqw fil-a, Alagwa fil-i, Burunge fil-u 'aardvark' [O. afer] | Qwadza fil-imbayo 'honey-badger' [Mellivora capensis].

- Cf. SED II No. 173; AADB 2528. Cf. Blažek 1994: 196.

\section{4. *gumar- *garyam-'hippopotamus'}

Chad.: C. *garyam-111 'hippopotamus' [Hippopotamus amphibius]: Musgu géryam, Vulum, Mbara gàriyàm, Masa gáryam, Banana garyam-ba \|| E. * girim-: Tumak gìrim, Birgit gìrím-tí.

Cush. * gumar-112 'hippopotamus' [H. amphibius]: ${ }^{113}$ C. * gumar-ī: Khamir gumär-i, Khamta

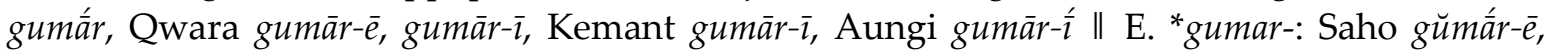
Afar gŭmá́r-ī | HEC: Qabenna gomarr-a, Tembaro gumorr-a. ${ }^{114}$

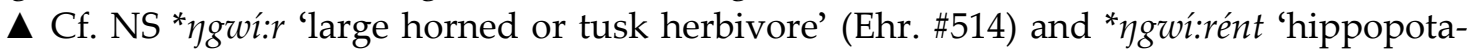
mus' (ibid. \#515).

AADB 2526; cf. Blažek 1994: 202-3.

\section{5. *labz- 'k. of largest herbivore'}

Egyp. irb? (<*2V-lbV?-?) $)^{115}$ 'rhinoceros (?)' [Diceros bicornis].

Chad. W. *lab-: Tangale lab-ata II E.: Mokilko Rêlb-í (met.?) 'elephant' [Loxodonta africana]. ${ }^{116}$

${ }^{109}$ Blench, 2009 :141 (draft printout); ngir ideof. describes large number of people or birds sitting in one place.

${ }^{110}$ Cf. also W. * pil: Gwandara pìil 'spear grass' [Heteropogoncontortus], Angas piil 'elephant grass' [Pennisetum purpureum]. It is an important element of forage for elephants in Africa, hence its name "elephant grass".

111 The metathesis is probably due to folk etymology, cf. Chad. C. *gar- 'bull': Musgu gari '(big) bull', Muskum gèrré, Vulum gàri'bull' and PAA *yVm- 'water'. Cf. Blažek 1994: 204.

112 Blažek also refers to Militarev's Ms. from 1987, in which Berb. *a-gumār 'horse' is compared to this Cush. term, referring to a well-known connection between horse and hippopotamus. However, the expected semantic development is reference to a hippo as "(river) horse" metaphorically; the reverse meaning shift is hard to imagine, which makes the Chad-Cush. comparison, already problematic because of metathesis, even less probable.

${ }^{113}$ Borrowed in Eth. as *gwamāri 'hippopotamus' (note also Gz. gamar, Amh. gumarr-e 'large monkey'). Blažek (1994: 202) quotes Gordon's interpretation of Ugr. gmr as 'a kind of animal capable of fighting ferociously' (Gordon, 1965: 380), calling it “a promising Semitic cognate”. In Olmo Lete and Sanmartín 2003, however, gmr is glossed as 'champion, fighter' with Sem. cognates.

${ }^{114}$ Cf. Omot. N.: Kullo gomár-a 'hippopotamus', isolated, likely a lw. from HEC.

${ }^{115}$ Otherwise < *2V-rbV?- comparable to E. Cush. *2arb- 'elephant'.

${ }^{116}$ Cf. Mokilko lòllib-ò 'trunk'. 
Cush. HEC *la?ab-(met.?): Hadiya, Kambatta loob-a 'hippopotamus' [Hippopotamus amphibius]. AADB 2513. Cf. HSED \#1662; Blažek 1994: 203.

3.6. * pir- 'k. of largest herbivore'

Sem.: Akk. (OB on) pìr-u, pēr-u 'elephant' [Loxodonta africana]. ${ }^{117}$

Chad. *pir-: W.: Hausa fyár-mà 'a young female elephant' || C.: Margi pir, Sukur n-ver-i $\left(<{ }^{*} n\right.$-per-i) 'elephant'. ${ }^{118}$

Cush. E. *pury-: Yaaku puria2, pl. puri-ain 'rhinoceros' [Diceros bicornis].

AADB 4259.

\section{7. * war $\hat{s}$ - 'rhinoceros'119}

Sem.: Eth. *hawriŝ-' 'rhinoceros' [Diceros bicornis]: ${ }^{120}$ : Gz. hari $\hat{s}^{121}$ Tna., Tgr. hariš, Amh. haris, awraris. ${ }^{122}$

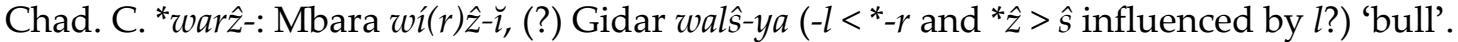

Cush. E. *worš- 'rhinoceros' [Diceros bicornis]: LEC *worš-: Oromo worse-sa, ${ }^{123}$ Konso orš-ayta (also 'hippo' [Hippopotamus amphibius] acc. to Black), Gidole orš-ayt, Dirayta orš-ayt (also 'gnu' [Connochaetes sp.]) I HEC *worš-a Gollango, Harso oršas-ado I Yaaku órse?.

$\Delta$ The resemblance of all these forms can hardly be accidental, though the origin of Eth. ${ }^{*} h$ - is far from clear. It could have been explained by ${ }^{*} h->\varnothing$ - in some of the non-Semitic languages quoted, but * $h$ is expected to be preserved as $h$ or $h$ in Hadiya, Gollango and Yaaku. It could be suggested that the Eth. term may go back to the compound form *hoyāw 'alive, living'124 + *wariŝ- paralleling Zarwehoras 'animal / beast + rhinoceros' > Zawrāhars > Zawrāris (above). Cf. especially Proto-North Caucasian *wHār גָwa 'pig, piglet'.

AADB 2529; cf. Blažek 2003b: 270, Blažek 1994: 201.

\section{8. *̌̆ag( $\left(^{w}\right)$ - 'k. of largest herbivore' ${ }^{125}$}

Egyp. (MK) z’g.t (met. <*2zg or ? renders -a-?) 'ein Fabeltier'.

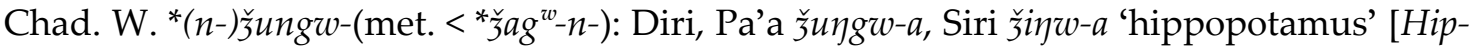
popotamus amphibius], Dwot $n$-dzugh-u 'elephant' [Loxodonta africana].

Cush. E. *zag-am-: Yaaku sogóm-èi 'elephant' [L. africana]. ${ }^{126}$

Omot. N. *2a-zag-ay 'hippopotamus' [H. amphibius]: Kačama azāgēe, Koyra azzāgēe, azzagi, Ganjule azagé.

117 Usually regarded as a by-form of $p \bar{l}-u$ (see in 3.3) due to its sound Sem. etymology, indisputable in light of Gz. falfal (cf. discussion in SED II: 228).

${ }^{118}$ Cf. W.: Kofyar feer, Warji farai, párái, Karya pár, Miya à-pár 'horn'; C.: Daba fàlām (-l can be <*-r), Buwal fár-ám, etc. 'horn'.

119 The reconstruction of ${ }^{*}-\hat{s}\left(\right.$ not $\left.^{*}-\hat{c}\right)$ is based on the Mbara form alone.

120 There is also Syr. hars- $\bar{u} m-\bar{a}$ 'proboscis; labia bovis' which matches the Eth. noun phonetically, but semantically seems not close enough to be related.

${ }^{121}$ Also ḥaris, hִoras, Zarwehoras, Zawrāhars, Zawrāris.

${ }^{122}$ Arab. harī̌s- is a lw. < Gz.; E. Cush.: Saho hariš < Mod. Eth.

$123<$ *worse-ta; cf. Bayso wōrsēsa, borrowed from Oromo.

$124<$ Sem. *hyw/y 'to live' (> Hbr. hayyā 'animals, beasts', Syr. hayyūt-, Hbt. haywuún 'animal').

${ }^{125}$ Cf. the similar SAA root *̌̌akan- 'elephant' [L. africana]: Cush. E.: Saho, Afar dakaan-o (< *dakan- or *̌̌akān-), LEC: Somali dagon (<*dakVn- or *̌̌akVn-), Dahalo dokom-i, dokom-i (<*dVkVm- or, perhaps, *̌̌ VkVm-; the Dahalo reflex of AA *̌̌ is not clear - see Takács 2011: 119); Omot. N. *zak(kV)-nV: Kačama, Koyra zākk-āa, Gofa zakkk-i, Ganjule $z \underline{a k k-a}$, Yemsa $z a k n-\bar{o}$ (the Omot. terms may otherwise have been borrowed from Bantu *nzok 'elephant').

${ }^{126} s$ in Yaaku may reflect ${ }^{*} z<{ }^{*} / \check{3}$. 
AADB 2521. Cf. HSED 2658; Blažek 1994: 204.

\section{9. *̌̌uną- 'elephant'}

Chad. *žunar- 'elephant' [Loxodonta africana]: W. *žawn-: Ngizim žàun-àk \|| C. *̌̌uwan-: Tera క̌ùwàn, Sukur క̌uwan I| E.: *̌̌una?: Nanchere žena?, Gabri క̌én-u, Kabalai, Dormo žun-o.

Cush. *̌̌uną- 'elephant' [L. africana]: C. *̌̌aHun- (met.): Bilin, Qwara, etc. $\breve{z} \bar{a} n-\bar{a}$, Khamir

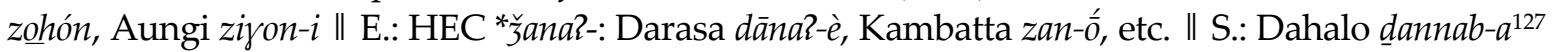
'female elephant'.

- AADB 2522. Cf. HSED \#2658; Blažek 1994: 199.

\section{Discussion}

Given the above genealogical classification of Afrasian, only those roots whose reflexes are attested in both SEBC and CO branches can be labeled PAA; attestation in Semitic makes this status incontestable. In case of faunistic terms, though, this principle cannot be strictly followed, since the retention of PAA animal names depended on availability of the denotata in the area where the respective language speakers had migrated.

There are two opposing views on the ancestral home of the Afrasians. Alexander Militarev's Levantine hypothesis is supported by Blažek (2013), and a similar view is held by the archaeologist Peter Bellwood (2013). The idea of an African homeland, originally put forward by Igor Diakonoff (1981; later, however, he would lean more towards the Levantine hypothesis, cf. Diakonoff 1998) is promoted in various works by Christopher Ehret (e.g., Ehret 2011) and Roger Blench (e.g., Blench 2006) and, to a certain degree, shared by George Starostin (Starostin 2017).

The hypothesis according to which Proto-Afrasians may be associated with the highly developed Natufian and Post-Natufian Epipaleolithic culture of hunters and gatherers $(12,500-9,500$ BCE) is based on several main arguments. First, some of the multiple reconstructed terms ${ }^{128}$ point to incipient agriculture rather than foraging. ${ }^{129}$ For such an early period, both are typical of West Asia, the South Levant in particular, rather than of East or North Africa. Second, the whole picture of PAA culture reconstructed from the PAA lexicon (referring to social and economic differentiation, some terms relating to trade, weaponry, building and dwelling, etc.) features a society highly developed for that period which, again, implies the Levant rather than East or North Africa. Third, there are at least several dozens of hypothetical cultural isoglosses between the non-Semitic Afrasian daughter proto-languages (Berber, Chadic, Cushitic) and, on one hand, Sumerian (Militarev 1995) and, on the other, North Caucasian (Militarev \& Starostin 2007), speaking in favor of ethnic and cultural contacts between

127 If analyzed as $\underline{d} a n n a-b a$, this would be another case of Dahalo $\underline{d}$ (usually $<* d$ ) as a reflex of $* \check{z} / 3$, besides másade 'in *maSi(n)z- 'k. of ungulate' (1.5b.)

${ }^{128}$ Ca. 30 in Militarev 2002; the number has more than doubled with a lot of newly processed data, especially Chadic - publication of a new list is forthcoming.

129 Since the $11^{\text {th }}$ millennium is regarded by modern scholarship as too early a period for incipient farming, the following scenario could explain the inventory of terms referring to farming and formally qualifying for ProtoAfrasian status: PSA speakers moved southward from the Levant to the north and, later, south Arabia (from where they moved to East Africa supposedly by or in the $8^{\text {th }}$ mill.), while PNA speakers stayed in the Levant and created the Pre-Pottery Neolithic A culture which set farming in motion in the $10^{\text {th }}$ mill.; some of the farming practices and terms could have passed from them to Proto-South Afrasians who were still located in Arabia, creating the illusion of Proto-Afrasian farming terminology when, in fact, it is representing only the PNA (PSEBC) stratum. 
the corresponding speaking groups which could take place in West Asia in the period between the split of PAA and migration of most of its daughter proto-languages to Africa. ${ }^{130}$

Counter-arguments rely on the repertoire of the PAA zoonyms denoting numerous African wild bovids (including most "antelopes"), elephants, rhinoceroses, hippopotami, crocodiles and odd-toed ungulates (except for onager) and large carnivores (lion, leopard, wolf, jackal, etc.). African animals did not inhabit the Levant during the warmer Preboreal phase of Holocene, which includes the Pre-Pottery Neolithic period (ca. 9,500-8,500 BCE, see Table 1), though the names of these animals are technically reconstructed on the PAA level. Regular consonantal correspondences between the reflexes of these roots point to their PAA origin, though the primary meaning of PAA terms is hard to specify considering the length of time that has passed since the PAA split.

Climate of the Levant in the era of the Natufian culture (ca. 12,500-9,500 BCE) was unacceptable for tropical mammals. The Natufians hunted only the following ungulates: gazelles [Gazella gazella, G. subgutturosa], deer [Cervus elaphus, Damadama], ibex [Capra ibex], aurochs [Bos primigenius], wild boar [Sus scrofa] and onager [Equus hemionus]. ${ }^{131}$ A corresponding set of wild animal names has been reconstructed for Proto-Semitic (see SED II) whose Urheimat was most likely situated in West Asia, including the Levant, whereas for PAA as many as ca. 50 terms for wild ungulates have been reconstructed, most of them presumably representing various species. Such a variety of ungulate species is typical of the North East African subequatorial fauna, which has essentially remained unchanged since the end of Pleistocene.

Many terms denoting tropical African "antelopes"132 and largest animals such as elephant, hippopotamus, and rhinoceros, are to be formally considered PAA as well. These PAA roots are usually well reflected in Chadic (less so in Berber and Egyptian), Cushitic, and/or Omotic, but poorly attested in Semitic, whose original habitat being West Asia, the terms for "largest herbivores" sometimes refer to mythical animals ('monster', 'fabulous bull' and the like), while those for other ungulates usually denote various species of cattle, sheep and goats, asses, ${ }^{133}$ horses and camels.

Another group of PAA zoonyms relevant for the Urheimat problem is represented by predators. There are 8 reconstructed terms for canines including 'dog' ( ${ }^{*} k a w i t-,{ }^{*} k^{w i h a n-}$,

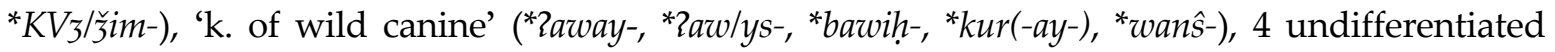

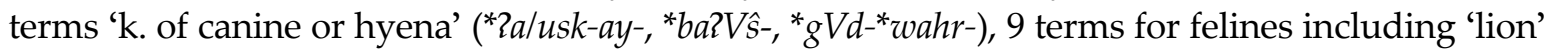

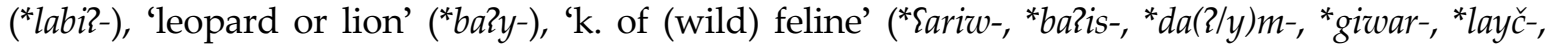
${ }^{*}$ mary-, *sawr-), and one undifferentiated term 'k. of feline, hyena or viverra' (*2ažur-). ${ }^{134}$ These considerations should rather stimulate searching for the PAA homeland in the East African area, a hypothesis already voiced by several scholars, including Igor M. Diakonoff and others.

${ }^{130}$ As for Sumerian-Afrasian (non-Semitic) suggested isoglosses, all of them have a PAA origin and, on chronological grounds, can only be loanwords into Sumerian from various intermediate proto-languages of different Afrasian branches. It is possible that another (extinct and unattested) branch of AA in Mesopotamia could function as a substratum under Sumerian; this assumption by Militarev (1995) was accepted as probable by Diakonov (1996).

131 See Mithen 2003; Lev et al. 2020.

132 "Antelopes" comprise a miscellaneous group within bovids, encompassing Old World (mostly African) ruminants that are not bovines, sheep, or goats.

${ }^{133}$ In this connection it is worth mentioning that the only species of odd-toed ungulates attested in the Levant is the onager, whereas Africa was home to several species of wild equids.

134 The detailed elaboration of these terms is prepared for publication by the same authors as well as reconstruction of other PAA zoonyms including monkeys (4 terms - none in Semitic), other mammals (8 terms, a selection), birds (11, a selection), reptiles (9, a selection), water reptiles and amphibia (4, a selection), fish (5, a selection). 

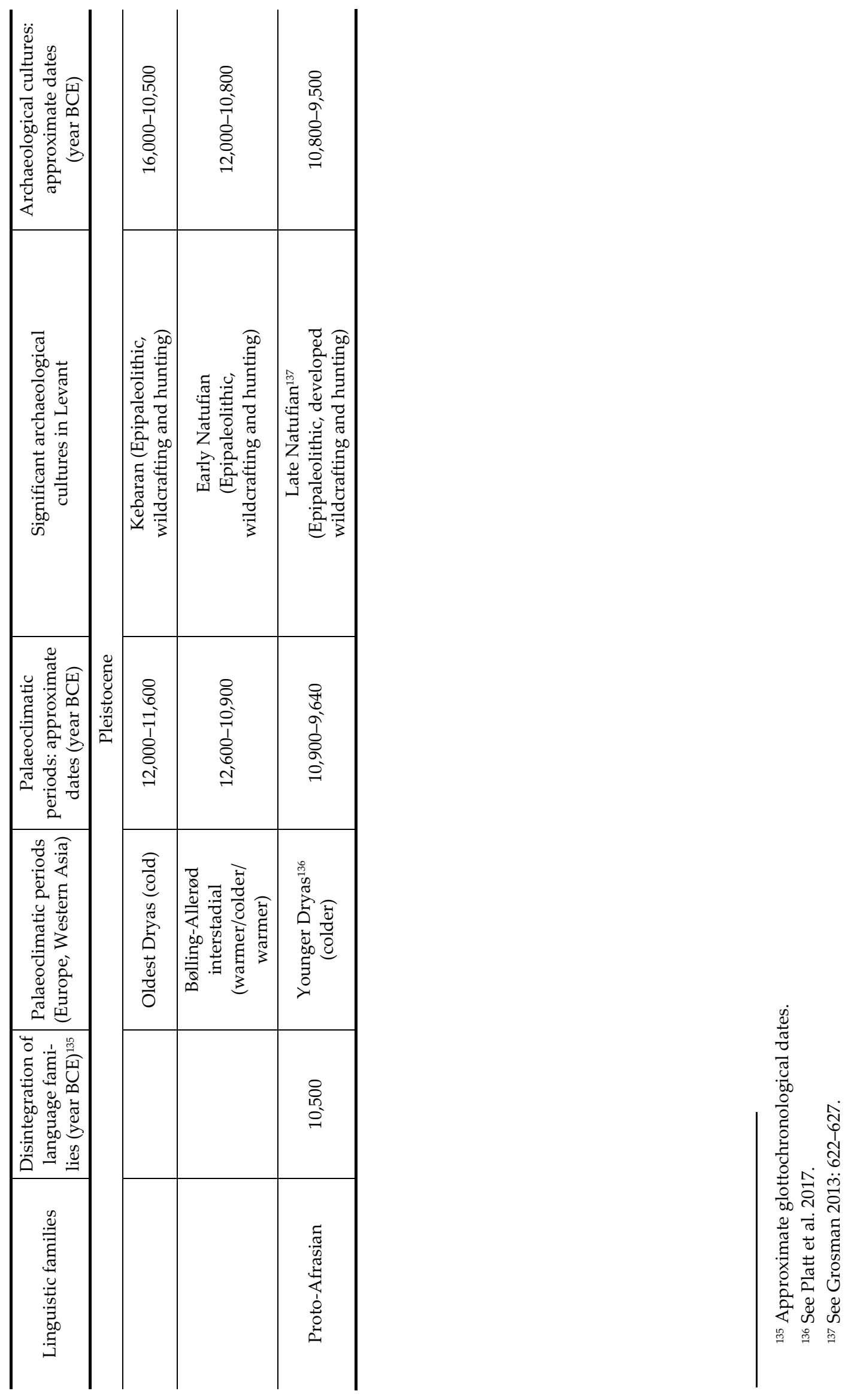


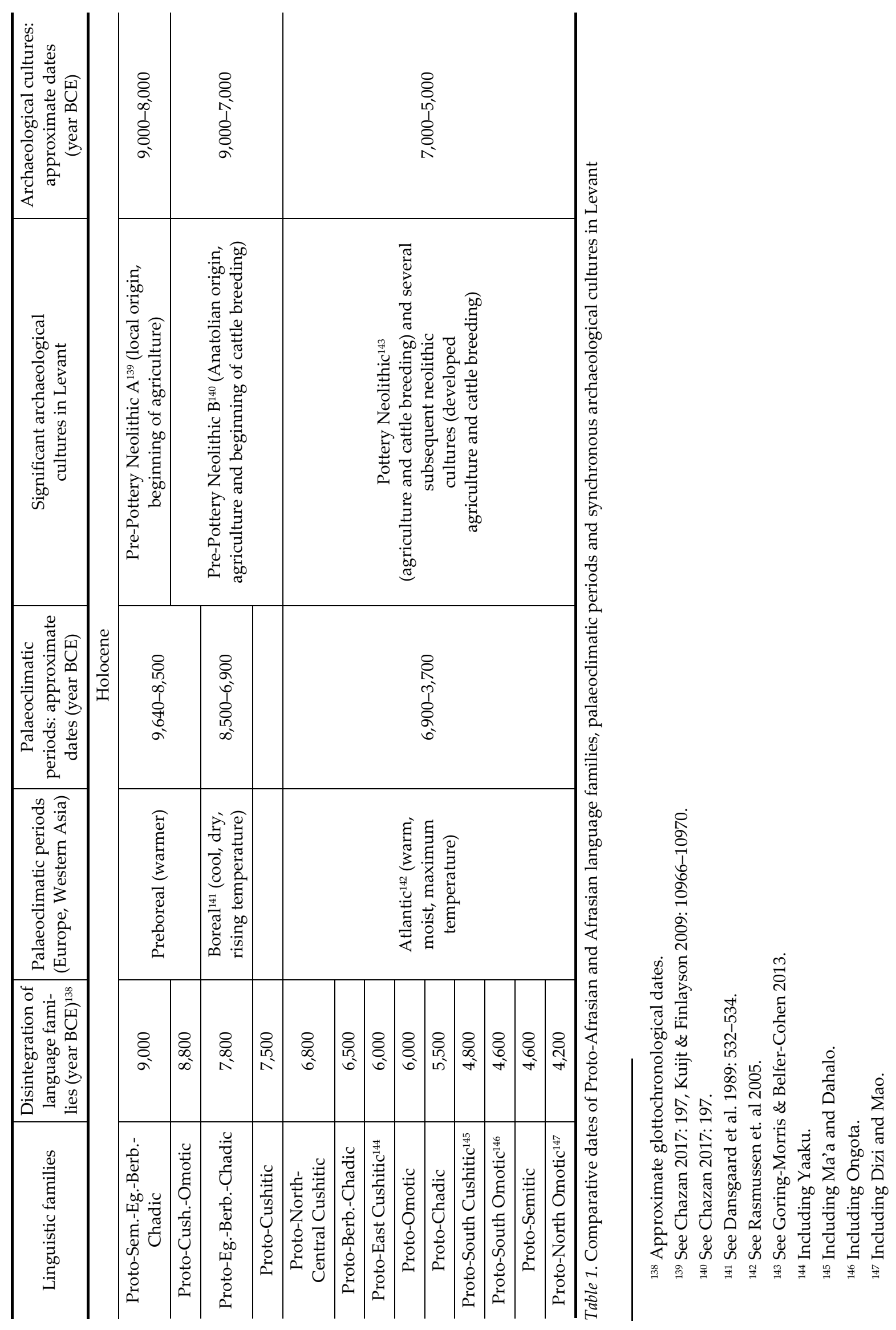


Solid retention of PAA roots denoting representatives of African fauna in Proto-Chadic contrasts with weaker integrity in Egyptian and Proto-Berber and largely relict reflexation in Proto-Semitic. This may be explained by the latitudinal arrangement of the proto-languages descending from PSEBC dialects. The aggregate area of the latter probably stretched from Northeast Africa up to the Levant. The Cushitic and Omotic homelands would be situated in East Africa further to the south than the PSEBC homeland.

\section{Appendix: Alphabetic index of reconstructed roots}

*2ar(a)w/y- 'k. of larger bovid' 1.1a.

*2ar(a)w/y- 'k. of lesser bovid'1.1b.

*2ayl- 'k. of lesser bovid' 1.2.

* aafVw- 'k. of lesser bovid' 1.3 .

* $\{i(n) 3-$ and *2i(n)3- 'k. of smaller bovid' 1.4a.

*ba/iz- *barba?- 'k. of lesser bovid (Tragelaphus silvaticus?)' 1.5 .

*balay/w- 'k. of lesser bovid' 1.6.

*baray- *bayar- 'k. of lesser bovid' 1.7 .

*cawiz- 'k. of bovid' 1.8 .

*čąiw- 'k. of lesser bovid' 1.9a.

*čawir- 'male large bovid or herbivore' 1.9b.

*da $(n) g^{w}(V r)$ - 'elephant' 3.1.

*dawk- 'k. of equid' 2.1.

*duhr- 'elephant' 3.2.

*(2a-)dury- 'k. of bovid' 1.10 .

${ }^{*} f a(2) n$ - 'k. of large bovid' 1.11 .

*fil- 'a proboscidean mammal' 3.2.

*(2a-)fir- 'k. of bovid' 1.12 .

*ga/ud- 'wild pig' 1.13b.

*gawr- 'k. of bovid' 1.14a.

* gayl- 'camel' 2.2.

* guday- 'k. of larger bovid' 1.13a.

*gumar- *garyam- 'hippopotamus' 3.4.

*gur- 'wild pig' 1.14b.
* yawz- 'k. of smaller bovid' 1.15.

*ha/ir- 'wild ass' 2.4.

*hawr- 'k. of larger bovid' 1.16 .

*hilu(l)l- 'k. of equid' 2.3.

${ }^{*} k^{w}$ ayr- 'young (small) bovid' 1.17.

${ }^{*} k^{w} a r$ - 'wild ass or camel' 2.5.

*labz- ' $\mathrm{k}$. of largest herbivore' 3.5.

*lawiz- 'k. of large bovid' 1.18.

*ma-\{i(n)3- 'k. of bovid' 1.4b.

*mayn- 'k. of large bovid' 1.19 .

*mar(ay)- 'k. of large bovid' 1.20a

*(2a-)mayr- 'k. of small bovid' 1.20b.

*naRiw- 'k. of bovid' 1.21.

* pir- 'k. of largest herbivore' 3.6.

*pira?- 'k. of equid' 2.6.

*saraw/y- 'k. of bovid' 1.22.

*ŝaz- *ŝas- 'k. of large bovid' 1.23.

*wasil- 'k. of lesser bovid' 1.24.

*wad- 'k. of lesser bovid' 1.25.

*(2a-)w/yan- 'k. of lesser bovid' 1.26.

*war- 'k. of larger bovid' 1.27.

*warŝ- 'rhinoceros' 3.7.

${ }^{*} \breve{a} a g^{(w)}$ - 'k. of largest herbivore' 3.8 .

*ร̌una?- 'elephant' 3.9.

\section{Abbreviations}

Names of languages

$\begin{array}{llllll}\text { AA } & \text { Afrasian =Afro-Asiatic } & \text { Copt. } & \text { Coptic } & \text { ESud } & \text { East Sudanic } \\ \text { Akk. } & \text { Akkadian } & \text { Cush. } & \text { Cushitic } & \text { Eth. } & \text { Ethiopic } \\ \text { Amh. } & \text { Amharic } & \text { Dem. } & \text { Demotic } & \text { Ez. } & \text { Eza } \\ \text { Arab. } & \text { Arabic } & \text { E. Wlm } & \text { East Tawllemmet } & \text { Gog. } & \text { Gogot } \\ \text { Aram. } & \text { Aramaic } & \text { Ebl. } & \text { Ebla } & \text { Gt. } & \text { Gyeto } \\ \text { Berb. } & \text { Berber } & \text { Egyp. } & \text { Egyptian } & \text { Gur. } & \text { Gurage } \\ \text { Bibl. } & \text { Biblical Aramaic } & \text { End. } & \text { Endegen } & \text { Gz. } & \text { Ge'ez } \\ \text { Ch. } & \text { Chaha } & \text { Enn. } & \text { Ennemor } & \text { Har. } & \text { Harari } \\ \text { Chad. } & \text { Chadic } & \text { ESA } & \text { Epigraphic South Arabian } & \text { Hbr. } & \text { Hebrew }\end{array}$




\begin{tabular}{|c|c|c|c|c|c|}
\hline $\mathrm{Hbt}$ & Hobyoț & NE & New Kingdom & Sab. & Sabaic \\
\hline HEC & Highland East Cushitic & NS & Nilo-Saharan & Sam. & Samaritan \\
\hline Hrs. & Harsusi & OAkk & Old Akkadian & Sel. & Selti \\
\hline Jib. & Jibbali & OB & Old Babylonian & Sem. & Semitic \\
\hline Jud. & Judaic Aramaic & Off. & Official Aramaic & SNil & South Nilotic \\
\hline LEC & Lowland East Cushitic & $\mathrm{OK}$ & Old Kingdom & Soq. & Soqotri \\
\hline Mand. & Mandaic & PAA & Proto-Afrasian & Sum. & Sumerian \\
\hline MB & Middle Babylonian & $\mathrm{PCO}$ & Proto-Cushitic-Omotic & Syr. & Syriac \\
\hline Mhr. & Mehri & Phoen. & Phoenician & Tgr. & Tigre \\
\hline Min. & Minaean & PSEBC & Proto-Semitic-Egyptian- & Tna. & Tigrinya (Tigra) \\
\hline MK & Middle Kingdom & & Berber-Chadic & Ugr. & Ugaritic \\
\hline Ms. & Masqan & Qat. & Qatabanian & Wol. & Wol. \\
\hline MSA & Modern South Arabian & S.Cush. & Southern Cushitic & YB & Young Babylon \\
\hline \multirow[t]{2}{*}{ Muh. } & Muher & SA & Saho-Afar & & \\
\hline & & & Terminology & & \\
\hline coll. & collective & k. & kind & redupl. & reduplication \\
\hline dial. & dialect & lw. & loanword & $\mathrm{m}$. & masculine \\
\hline dissim. & dissimilation & met. & metathesis & sg. & singular \\
\hline f. & feminine & pers. & personal & sp. & species \\
\hline gen. & generic (term) & pl. & plural & subst. & substantive \\
\hline$i d$ & idem & pref. & prefix & suff. & suffix \\
\hline
\end{tabular}

Other notational symbols

\| separates branches within a language family

I separates subbranches or groups

- denotes morphemic boundaries ${ }^{148}$

Abbreviations of sources

AADB $=$ Militarev \& Stolbova 2020

AHw = von Soden 1965-1981

BK $=$ Biberstein-Kazimirski 1860

CAD $=$ Brinkman et al. 1956-2010

$\mathrm{CED}=$ Stolbova 2016
DRS = Cohen et al. 1970-1993

EDE $=$ Takács 1999, 2001, 2008

EDS $=$ Parpola 2015

HSED = Orel \& Stolbova 1995

Mil. 2009 = Militarev 2009

SED II = Militarev \& Kogan 2005

\section{References}

Appleyard, David. 2006. A Comparative Dictionary of the Agaw Language. Köln: Rüdiger Köppe.

Bassano, Francesco da. 1918. Vocabolario tigray-italiano e repertorio italiano-tigray. Roma: Casa editrice italiana \& C. de Luigi.

Beeston, Alfred F. L., Mahmud Ali Ghul, Walter W. Müller, Jacques Ryckmans. 1982. Sabaic Dictionary. Louvain-laNeuve, Éditions Peeters; Beyrouth, Librairie du Liban.

Bellwood, Peter. 2013. First Migrants. Ancient Migration in Global Perspective. Chichester: Wiley - Blackwell.

Biberstein-Kazimirski, Albert de. 1860. Dictionnaire arabe-français. Vol.1-2. Paris: Maison-Neuve et Co.

Biella, Joan C. 1982. Dictionary of Old South Arabic. Sabaean Dialect. Harvard Semitic Studies, 25. Chico (CA): Scholars Press.

${ }^{148}$ In some cases, especially in Chadic lexemes, boundaries drawn between the stem and affixes and between the affixes may not be exact, since ideal morphemic analysis requires more detailed knowledge of each individual language's morphology and morphophonemics than possessed by the authors. 
Bittner, Maximillian. 1917. Studien zur Shauri-Sprache in den Bergen von Dofar am Persischen Meerbusen. Wien: Hölder. Blažek, Václav. 1994. Elephant, Hippopotamus and Others: On Some Ecological Aspects of the Afroasiatic Homeland. Asian and African Studies 3/2: 196-212.

Blažek, Václav. 2003a. Semitic táwar- 'Bull' and Its Relatives. In: M. L. Bender, D. Appleyard, G. Takács (eds). Selected Comparative-Historical Afrasian Linguistics Studies in Memory of Igor M. Diakonoff: 7-12. München: Lincom Europa.

Blažek, Václav. 2003b. Fauna in Beja Lexicon. In: Ilya Smirnov (ed.). Studia Semitica: Fs. for Alexander Militarev: 230294. Moscow: Russian State University for the Humanities.

Blažek, Václav. 2013. Levant and North Africa: Afroasiatic linguistic history. In: Peter Bellwood (ed.). The Encyclopedia of Global Human Migration. Vol. 1. Prehistory: 125-132. London: Blackwell.

Blench, Roger. 2006. Archaeology, Language, and the African Past. Lanham: Altamira Press.

Blench, Roger. 2009. A Dictionary of Bura. Available at: www.rogerblench.info/Language/Afroasiatic/Chadic/Central/ Bura/Bura\%20dictionary\%202010.pdf [Accessed 28.12.2020].

Brinkman, John A., Miguel Civil, Ignace J. Gelb, A. Leo Oppenheim, Eruca Reiner (eds.). 1956-2010. The Assyrian Dictionary of the Oriental Institute, the University of Chicago. Chicago, Illinois, U.S.A.: Oriental Institute; Glückstadt, Germany: J.J. Augustin Verlagsbuchhandlung.

Chazan, Michael. 2017. World Prehistory and Archaeology: Pathways Through Time. 4th edition. London: Routledge.

Cohen, David, et al. 1970-1993. Dictionnaire des racines sémitiques ou attestées dans les langues sémitiques. Fasc. 1-2. Paris: Mouton. Fasc. 3-5. Leuven: Peeters.

Conti Rossini, Carlo. 1913. Schizzo del dialetto Saho dell'Alta Assaorta in Eritrea. Roma, Tip. della R. Accademia dei Lincei. V. XXII, Fasc. 5.

Dansgaard, Willi, James W. C. White, Sigfús J. Johnsen. 1989. The abrupt termination of the Younger Dryas climate event. Nature 339: 532-534.

Diakonoff, Igor M. 1981. Earliest Semites in Asia: agriculture and animal husbandry according to linguistic data (VIIIth-IVth milennia). Altorientalische Forschungen 8: 23-74.

Diakonov, Igor M. 1996. Shumery i afrazijcy glazami istorika [Sumerians and Afrasians through the eyes of an historian]. Vestnik Drevnej istorii 4: 81-88.

Diakonoff, Igor M. 1998. The earliest Semitic society. Linguistic data. Journal of Semitic Studies 43 (2): 209-219.

Dolgopolskiy, Aaron B. 1973. Sravnitel'no-istoričeskaja fonetika kušitskix jazykov [Comparative-Historical Phonetics of the Cushitic Languages]. Moskva: Nauka.

Ehret, Christopher. 1980. The Historical Reconstruction of Southern Cushitic Phonology and Vocabulary. Berlin: Dietrich Reimer Verlag.

Ehret, Christopher. 2001. A Historical-Comparative Reconstruction of Nilo-Saharan. Köln: Rüdiger Köppe.

Ehret, Christopher. 2011. History and the Testimony of Language. Berkeley: University of California Press.

Erman, Adolf, Hermann Grapow. 1957-1971. Wörterbuch der aegyptischen Sprache. I-V. Berlin: Akademie-Verlag

Grosman, Leore. 2013. The Natufian Chronological Scheme - New Insights and their Implications. In: Ofer BarYosef, François R.Valla (eds.). Natufian Foragers in the Levant: Terminal Pleistocene Social Changes in Western Asia: 622-637. New York: Berghahn Books.

Johnstone, Thomas M. 1987. Mehri Lexicon and English-Mehri Word List. London / New York: Routledge.

Jungraithmayr, Herrmann, Dymitr Ibriszimow. 1994. Chadic Lexical Roots. Vols. I-II. Berlin: Dietrich Reimer Verlag.

Goring-Morris, A. Nigel, Anna Belfer-Cohen. 2013. The Southern Levant (Cisjordan) During the Neolithic Period. In: Ann E. Killebrew, Margreet Steiner (eds.). The Oxford Handbook of the Archaeology of the Levant: c. 8000-332 BCE: 141-163. Oxford University Press.

Kuijt, Ian, Bill Finlayson. 2009. Evidence for food storage and predomestication granaries 11,000 years ago in the Jordan Valley. In: Proceedings of the National Academy of Sciences of the United States of America 106 (27): $10966-$ 10970.

Lanfry, Jacques. 1973. Ghadamès. Vol. II. Glossaire (Parler des Ayt Waziten). Algérie: Fort-National, Fichier de documentation berbère.

Leslau, Wolf. 1987. Comparative Dictionary of Ge'ez (Classical Ethiopic). Wiesbaden: Otto Harrassowitz.

Lev, Ma'ayan, Mina Weinstein-Evron, Reuven Yeshurun. 2020. Squamate bone taphonomy: A new experimental framework and its application to the Natufian zooarchaeological record. Nature Scientific Reports 10. Available at: https://www.nature.com/articles/s41598-020-66301-5 [Accessed 28.12.2020].

Militarev, Alexander. 1995. Sumerians and Afrasians. Journal of Ancient History 2: 113-127. 
Militarev, Alexander. 2002. The prehistory of a dispersal: Proto-Afrasian (Afroasiatic) farming lexicon. In: Peter Bellwood, Colin Renfrew (eds.). Examining the Farming/Language Dispersal Hypothesis: 135-150. Cambridge: McDonald Institute for Archaeological Research.

Militarev, Alexander. 2005. Root extension and root formation in Semitic and Afrasian. Aula Orientalis 23(1): 83-130.

Militarev, Alexander. 2009. Proto-Afrasian Lexicon Confirming West Asian Homeland: Pastoralism. Journal of Language Relationship 1: 95-106.

Militarev, Alexander, Leonid Kogan. 2005. Semitic Etymological Dictionary. Vol. II: Animal Names. Münster: UgaritVerlag.

Militarev, Alexander, Olga Stolbova. 2020. Afroasiatic Etymology Database. Available at http://starling.rinet.ru [Accessed 28.12.2020].

Mithen, Steven. 2003. After the Ice: A Global Human History, 20,000-5000 BC. Cambridge, MA: Harvard University Press.

Motylinski, Adolphe de Calassanti. 1904. La dialecte berbère de R'edamès. Paris: E. Leroux.

Naït-Zerrad, Kamal. 1998, 1999, 2002. Dictionnaire des racines berbères. Paris-Louvain: Peeters Publishers.

Nakano, Akio. 1986. Comparative Vocabulary of Southern Arabic: Mahri, Gibbali and Soqotri. Tokyo: Institute for the Study of Languages and Cultures of Asia and Africa.

Olmo Lete, Gregorio del, Joaquín Sanmartín. 2003. A Dictionary of the Ugaritic Language in the Alphabetic Tradition. Leiden-Boston: Brill.

Orel, Vladimir, Olga Stolbova. 1995. Hamito-Semitic Etymological Dictionary. Leiden / New York / Köln: Brill.

Parpola, Simo. 2015. Etymological Dictionary of the Sumerian Language. Part 1. Lexical Evidence. Winona Lake, Indiana: Eisenbrauns.

Platt, Daniel E., Marc Haber, Magda Bou Dagher-Kharrat, Bouchra Douaihy, Georges Khazen, Maziar Ashrafian Bonab, Angélique Salloum, Francis Mouzaya, Donata Luiselli, Chris Tyler-Smith, Colin Renfrew, Elizabeth Matisoo-Smith, Pierre A. Zalloua. 2017. Mapping Post-Glacial expansions: The Peopling of Southwest Asia. Nature Scientific Reports 7: 40338. Available at: https://www.nature.com/articles/srep40338 [Accessed 28.12.2020].

Rasmussen, Sune O., Bo M. Vinther, Henrik B. Clausen, Katrine K. Andersen. 2005. The Greenland Ice Core Chronology 2005 (GICC05). Early Holocene section. IGBP PAGES/World Data Center for Paleoclimatology Data Contribution Series \# 2006-119. NOAA/NCDC Paleoclimatology Program, Boulder CO, USA.

Reinisch, Leo. 1890. Wörterbuch der Saho-Sprache. Wien: Alfred Hölder.

Reinisch, Leo. 1902. Die Somali-Sprache. Wörterbuch. Wien: Alfred Hölder.

Skinner, A. Neil. 1996. Hausa Comparative Dictionary. Köln: Rüdiger Köppe Verlag.

Soden, Wolfram von. 1965-1981. Akkadisches Handwörterbuch. Wiesbaden: Otto Harrassowitz.

Starostin, Sergei A. 2000. Comparative-historical linguistics and lexicostatistics. In: Colin Renfrew, April M. S. McMahon, Robert Lawrence Trask (eds.). Time Depth in Historical Linguistics. Vol. 1: 233-259. Cambridge: The McDonald Institute for Archaeological Research.

Starostin, George. 2017. Macrofamilies and agricultural lexicon. Problems and perspectives. In: M. Robbeets, A. Savelyev (eds.). Language Dispersal Beyond Farming: 215-233. Amsterdam / Philadelphia: John Benjamins.

Stolbova, Olga V. 2005. Chadic lexical database. Issue I: Letters L, N, NY, R. Moscow: Institute of Oriental Studies, Russian Academy of Sciences.

Stolbova, Olga V. 2009. Leksicheskaja baza dannyx po chadskim jazykam. V. 3.Sibil'anty i sibil'antnyje affrikaty. [Chadic Lexical Database. Issue III. Sibilants and Sibilant Affricates.] Moscow: Institute of Oriental Studies, Russian Academy of Sciences.

Stolbova, Olga V. 2011. Leksicheskaja baza dannyx po chadskim jazykam. V. 5.Vel'arnyje. [Chadic Lexical Database. Issue IV. Velars.] Moscow: Institute of Oriental Studies, Russian Academy of Sciences.

Stolbova, Olga V. 2016. Chadic Etymological Dictionary. Moscow: Institute of Oriental Studies of the Russian Academy of Sciences.

Stolbova, Olga V. 2019. Leksicheskaja baza dannyx po chadskim jazykam. V. 5. [Chadic lexical database. Issue V.]. Moscow: Institute of Oriental Studies, Russian Academy of Sciences.

Takács, Gábor. 1999. Etymological Dictionary of Egyptian. Vol. 1: A Phonological Introduction. Leiden / Boston / Köln: Brill.

Takács, Gábor. 2001. Etymological Dictionary of Egyptian. Vol. 2: B-, P-, F-. Leiden / Boston / Köln: Brill.

Takács, Gábor. 2008. Etymological Dictionary of Egyptian. Vol. 3: M. Leiden / Boston: Brill.

Takács, Gábor. 2011. Studies in Afro-Asiatic Comparative Phonology: Consonantism. Berlin: Dietrich Reimer Verlag. 
А. Ю. Милитарев, С. А. Николаев. Праафразийские названия копытных в свете проблемы афразийской прародины

В статье представлена первая порция полного тезауруса праафразийских зоонимов, подготовленного авторами: более ста названий копытных, включая полорогих, свиней, эквидов и верблюдов, а также крупных травоядных (слонов, гиппопотамов и носорогов), отражающие максимально полную (на современном уровне наших знаний) картину этих видов фауны в период накануне распада праафразийского языка (11 тыс. до н.э., по глоттохронологии) и в зоне обитания праафразийцев, две различные точки зрения на локализацию которой - Левант и Восточная Африка - также обсуждаются авторами.

Ключевые слова: афразийская прародина; афразийские (афро-азиатские) языки; этимология зоонимов; реконструкция культурной лексики. 